\title{
THREE DIMENSIONAL MECHANICAL MODEL TO SIMULATE THE NSM FRP STRIPS SHEAR STRENGTH CONTRIBUTION TO A RC BEAM: PARAMETRIC STUDIES
}

\author{
Vincenzo Bianco ${ }^{1}$, J.A.O. Barros ${ }^{2}$ and Giorgio Monti ${ }^{3}$
}

\begin{abstract}
This paper presents the results of a parametric study carried out with a a mechanical model recently developed to simulate the shear strength contribution provided by a system of Near Surface Mounted (NSM) Fiber Reinforced Polymer (FRP) strips applied to a Reinforced Concrete (RC) beam. That model, developed fulfilling equilibrium, kinematic compatibility and constitutive laws of both materials, concrete and FRP, and local bond between themselves, takes into consideration the possibility that the NSM strips may fail due to: loss of bond (debonding), concrete semi-conical tensile fracture or strip tensile rupture. The model also takes into consideration: a) interaction between progressive force transferred by bond to the surrounding concrete and its tensile fracture and b) bi-directional interaction among adjacent strips placed on the two sides of the strengthened beam cross-section web. In the first part of the paper attention is focused on the bond-based behavior of a single NSM FRP strip mounted on a concrete prism. The influence of each geometrical-mechanical parameter on the peak force transferable through bond stresses to the surrounding concrete, excluding the possibility of either concrete fracture and strip rupture, is analyzed. In the second part of the paper attention is focused on the comprehensive behavior of a single NSM FRP strip mounted on a concrete prism. The influence of each geometrical-mechanical parameter on the peak force transferable to the surrounding concrete, also including the possibility of both concrete fracture and strip rupture, is analyzed. The third part of the paper is devoted at assessing the influence of each geometrical-mechanical parameter on the maximum shear strength contribution provided by a system of NSM FRP strips applied to a RC beam. The results of these studies are presented along with the main findings.
\end{abstract}

Keywords: Numerical Model; FRP; NSM; Shear Strengthening; Concrete Fracture; Debonding; Tensile Rupture, Parametric Studies.

\section{Introduction}

Shear strengthening of RC beams by NSM technique consists in gluing FRP strips by an adhesive into thin shallow slits cut onto the concrete cover of the beam web lateral faces. This technique has been extensively investigated in recent

\footnotetext{
${ }^{1}$ Post Doctoral Fellow, Dept. of Structural Engrg. and Geotechnics, Sapienza University of Rome, via A. Gramsci 53, 00197 Rome, Italy. E-mail: vincenzo.bianco@uniroma1.it. Corresponding Author. Tel. +39 0649919254, Fax. +39063221449.

${ }^{2}$ Associate Professor, Dept. of Civil Engineering, University of Minho, Campus de Azurém, 4800-058 Guimarães, Portugal. E-mail: barros@civil.uminho.pt .

${ }^{3}$ Full Professor, Dept. of Structural Engrg. and Geotechnics, Sapienza University of Rome, via A. Gramsci 53, 00197 Rome, Italy. E-mail: giorgio.monti@uniroma1.it.
} 
years (e.g.: Mohammed Ali et al. 2006 and 2007, Bianco et al. 2006 and 2007, Dias et al. 2007, Dias and Barros 2008, Rizzo and De Lorenzis 2009; Dias and Barros 2011a,2011b). A three dimensional mechanical model was recently developed to simulate the NSM FRP strips shear strength contribution to a RC beam (Bianco 2008, Bianco et al. 2009ab). That model was developed fulfilling equilibrium, kinematic compatibility and constitutive laws of both materials FRP and concrete - and bond between themselves. From a physical point of view that model assumes that, during the loading process of a RC beam strengthened in shear by the NSM technique, the strips effectively crossing the Critical Diagonal Crack (CDC) oppose its widening by anchoring to the surrounding concrete to which they transfer, through bond stresses, the force originating at their intersection with the CDC. The relative movement of the two parts into which the CDC divides the beam web, imposes on the strips' available resisting bond lengths an increasing end slip (Bianco et al. 2009a). As function of the mechanical and geometrical properties characterizing a certain NSM shear strengthened RC beam, the failure mode assumed by each strip can be one of the following: a) complete extraction of the NSM FRP strip due to loss of bond throughout the strip available resisting bond length, in case where concrete mechanical properties are relatively high, which is, indeed, a very limit situation (debonding), b) concrete semi-conical fracture that reaches the strip free extremity (concrete semi-conical failure), c) concrete semi-conical fracture that stops progressing midway between loaded and free ends, with consequent debonding of the remaining portion of the available bond length (mixed-semi-cone-plus-debonding) and d) rupture of the strip independently of an initial concrete fracture (strip rupture). Note that the last three failure modes are brittle, while the first is more ductile (Bianco et al. 2009a). The mechanical model herein applied to carry out parametric studies takes also into account the possibility that strips placed on the two sides of the beam web can interact with each other. The analytical details of the mechanical model are herein omitted, for the sake of brevity, but they can be found elsewhere (Bianco 2008, Bianco et al. 2009a-b). The good predictive performance of this model was appraised on the basis of a large amount of experimental results (Bianco 2008).

In the first part of the paper, the model is applied to evaluate the influence of each geometrical-mechanical parameter on the peak force that a strip, near surface mounted on a concrete prism and subjected to an increasing imposed end slip, can transfer through bond stresses to the surrounding concrete. To that aim, the deformability of the concrete prism is accounted for, while the possibilities of either concrete fracture or strip rupture are excluded by assuming the tensile strength of both materials, concrete and FRP, infinitely large.

In the second part of the paper, the model is applied to evaluate the influence of each geometrical-mechanical parameter on the ultimate load that a single strip, near surface mounted on a concrete prism and subjected to an increasing imposed end slip, can comprehensively transfer to the surrounding concrete. For this purpose, the possibilities of occurrence of either concrete semi-conical tensile fracture or strip tensile rupture are accounted for. 
In the third part of the paper, the model is applied to evaluate the influence of each geometrical-mechanical parameter on the peak shear strength contribution provided by a system of NSM FRP strips to a RC beam.

\section{Bond-based behavior of a single NSM FRP strip subject to an imposed end slip}

Applying the model to the case of a single NSM FRP strip mounted on the surface of a concrete prism (Fig. 1), and neglecting the possibilities of either concrete semi-conical tensile fracture and strip rupture occur, it is possible to determine the bond-based constitutive law of the strip $V_{f i}^{b d}\left(L_{R f i} ; \delta_{L i}\right)$. This latter is the curve providing the bond-based force $V_{f i}^{b d}$ that the generic $i$-th strip, with resisting bond length $L_{R f i}$, can transfer, through bond stresses, to the surrounding concrete as function of the value of the increasing imposed end slip $\delta_{L i}$ (Fig. 1a-b). The bond-based constitutive law $V_{f i}^{b d}\left(L_{R f i} ; \delta_{L i}\right)$ of a given NSM FRP strip depends on the following parameters (Bianco et al. 2009a-b) (Fig. 1d-e): strip cross section thickness $a_{f}$ and width $b_{f}$; strip resisting bond length $L_{R f i}$; concrete prism cross section thickness $a_{c}$ and width $b_{c}$; concrete deformability $E_{f}$ (which is function of the concrete compressive strength $f_{c m}$ ); strip's Young's Modulus $E_{f}$ and values of bond stress $\left(\tau_{0}, \tau_{1}, \tau_{2}\right)$ and slip $\left(\delta_{1}, \delta_{2}, \delta_{3}\right)$ defining the local bond stress-slip relationship (Fig. 1e). The analytical details necessary to evaluate the constitutive law of a given NSM FRP strip are herein omitted, for the sake of brevity, but they can be found elsewhere (Bianco et al. 2009a-b). The constitutive laws $V_{f i}^{b d}\left(L_{R f i} ; \delta_{L i}\right)$ of NSM FRP strips of different values of $L_{R f i}$ can be plotted both in a bi-dimensional $\left(V_{f i}^{b d} ; \delta_{L i}\right)$ and in a three-dimensional $\left(V_{f i}^{b d} ; L_{R f i} ; \delta_{L i}\right)$ Cartesian orthogonal reference system. The peak bond-transferred force $V_{f i}^{b d, m a x}$ increases, by increasing $L_{R f i}$, up to the value $V_{f i}^{b d, \max }\left(L_{R f e}\right)$ corresponding to the effective resisting bond length $L_{R f e}$, which is the value of $L_{R f i}$ beyond it any further increase of the resisting bond length $L_{R f i}$ does not yield any further peak load gain. In a bi-dimensional representation (Fig. 1a), the point $\left(V_{f i}^{b d} ; \delta_{L i}\right)$ representative of the state of the NSM strip of resisting bond length $L_{R f i}$ moves, for increasing values of $\delta_{L i}$, on the same branch, non-linear ascending or linear horizontal for values of $L_{R f i} \leq L_{R f e}$ or $L_{R f i}>L_{R f e}$, respectively, common for each value of $L_{R f i}$, as long as $L_{R f i}$ is larger or equal to the value of the necessary bond transfer length $L_{t r}^{b d}\left(\delta_{L i}\right)$ (Bianco et al. 2009a-b). The $L_{t r}^{b d}\left(\delta_{L i}\right)$, which is function of $\delta_{L i}$ only, is the bond length that would be necessary to entirely transfer to the surrounding concrete a force equal to the one originating in the strip loaded end, due to the imposition of $\delta_{L i}$ (Bianco et al. 2009a). When the constitutive laws $V_{f i}^{b d}\left(L_{R f i} ; \delta_{L i}\right)$ of NSM FRP strips of different and increasing values of resisting bond length are represented in a three dimensional reference system, they form a continuous surface (Fig.1b). 
The parametric study presented in this paragraph concerns the influence of each parameter on the peak bond-transferred force for different values of $L_{R f i}$. Note that for each parameter was considered a range comprehending values a little beyond those having a strict physical confirmation, in order to assess not only their influence on the physical behavior of an NSM FRP strip, but also their influence from a mere analytical-numerical standpoint (Table 1). The results of the parametric studies are reported in the following.

A variation of the concrete Young's Modulus $E_{c}$ does not yield any variation of $V_{f i}^{b d \text {,max }}$, for whatever value of $L_{R f i}$ (Fig. 2a). An increase of the FRP Young's Modulus $E_{f}$ yields a resisting-bond-length-dependent increase of $V_{f i}^{b d \text {,max }}$

(Fig. 2d). In fact, $V_{f i}^{b d \text {, max }}$ increases with $E_{f}$, with a rate that is as higher as larger is the value of $L_{R f i}$. Moreover, the curves providing $V_{f i}^{b d, \text { max }}$ as function of $E_{f}$ and $L_{R f i}$ show two phases: a first one, coincident for all of the values of $L_{R f i}$, for which an increase of $E_{f}$ can be better felt since $L_{R f i}$ is larger than the corresponding value of the effective resisting bond length $L_{R f e}\left(E_{f}\right)$ and a second one, different for all of the values of $L_{R f i}$, in which an increase of $E_{f}$ can less be felt since $L_{R f i}$ is smaller than $L_{R f e}\left(E_{f}\right)$.

An increase of the strip cross section dimensions, either $a_{f}$ or $b_{f}$, yields a resisting-bond-length-dependent increase of $V_{f i}^{b d, \text { max }}$. In particular, for the case of the strip cross section thickness $a_{f}$, the curves providing $V_{f i}^{b d \text {,max }}$ as function of $a_{f}$, for different values of $L_{R f i}$, are superimposed as long as it is $L_{R f i} \geq L_{R f e}\left(a_{f}\right)$ and present a different increasing rate, as larger $L_{R f i}$ is, for values of $L_{R f i}<L_{R f e}\left(a_{f}\right)$.

A variation of the prism cross section dimensions, either $a_{c}$ or $b_{c}$, does not yield any appreciable variation of $V_{f i}^{b d, \text { max }}$, for whatever value of $L_{R f i}($ Fig. 2c,f).

The peak bond-transferred load $V_{f i}^{b d, \text { max }}$ increases by increasing the resisting bond length up to a certain value beyond which any further increase does not produce any further gain in terms of resistance (Fig. 1c). That threshold value of length, according to the terminology already adopted for the Externally Bonded Reinforcement (EBR), is herein labeled as effective resisting bond length $L_{R f e}$. Note that for the values of the mechanical parameters herein adopted (Table 1), $L_{R f e}$ is equal to $486 \mathrm{~mm}$.

As regards the influence of the parameters characterizing the local bond stress-slip relationship, it arises that $V_{f i}^{b d, \text { max }}$ is marginally affected when a realistic range of $\tau_{0}$ values is considered, for whatever value of $L_{R f i}$ (Fig. 3a). The peak bond-transferred force $V_{f i}^{b d \text {,max }}$ increases with a resisting-bond-length-dependent-rate by increasing the value of either 
$\tau_{1}$ or $\tau_{2}$. In particular, $V_{f i}^{b d, \text { max }}$ increases by increasing $\tau_{1}$ with a rate that is larger as shorter is the value of $L_{R f i}$, while $V_{f i}^{b d, \text { max }}$ increases with $\tau_{2}$ with a rate that is higher as larger is the value of $L_{R f i}$ (Fig. 3 b-c). $V_{f i}^{b d, \text { max }}$ decreases for increasing values of $\delta_{1}$ with a resisting-bond-length-dependent-rate: in fact, the larger the value of $L_{R f i}$, the smaller is the rate with which $V_{f i}^{b d, \max }$ decreases for increasing values of $\delta_{1}$ (Fig. 3d).

An increase of either $\delta_{2}$ or $\delta_{3}$ (Fig. 3e-f) yields an increase of $V_{f i}^{b d \text {,max }}$ with a rate that is resisting bond length dependent. In particular, for the case of $\delta_{3}$, the curves providing $V_{f i}^{b d \text {,max }}$ as function of $\delta_{3}$, for the different values of $L_{R f i}$, are superimposed, presenting the same trend, as long as $L_{R f i}$ is larger or equal to $L_{R f e}\left(\delta_{3}\right)$.

Note that, for all of the parameters analyzed, the curves providing $V_{f i}^{b d \text {,max }}$ as function of the generic parameter for the various values of $L_{R f i}$ tend, by increasing the value of $L_{R f i}$, to an envelope curve. Moreover, for values of $L_{R f i}$ larger than a certain value, the curves providing $V_{f i}^{b d, \text { max }}$ as function of the generic parameter are all superimposed to the envelope one.

\section{Comprehensive behavior of a single NSM FRP strip subject to an imposed end slip}

The model was also applied to study the comprehensive behavior of a single NSM FRP strip, mounted on a concrete prism and subjected to an increasing imposed end slip. For this purpose, the possibilities of both concrete semi-conical tensile fracture and strip tensile rupture were also taken into account. Typically, according to the model herein adopted (Bianco 2008, Bianco et al. 2009a), increasing the imposed end slip to the single NSM FRP strip, co-axial and progressively larger semi-conical concrete fractures subsequently form around the strip since the very first load steps (Fig. 4b,c). Contextually at the occurrence of each concrete semi-conical fracture, the initial value of the resisting bond length $L_{R f i}^{0}$ progressively reduces. Moreover, due to the formation of successive co-axial semi-conical fracture surfaces around the strip, the resulting concrete fracture envelope surface, which corresponds to the last and larger semi-conical surface, starting from the loaded end, progressively penetrates within the concrete prism (Fig. 4c). It can happen that the vertex of the last semi-conical concrete fracture surface places midway between the loaded and free end or reaches this latter. Moreover, contextually at the occurrence of each of those concrete fractures, the point representative of the state of the strip, with coordinates $\left(V_{f i} ; L_{R f i} ; \delta_{L i}\right)$ leaps, for a constant value of $\delta_{L i}$, from the bond-based constitutive law $V_{f i}^{b d}\left(L_{R f i}^{n} ; \delta_{L i}\right)$ of the previous value of resisting bond length $L_{R f i}^{n}$ to the one corresponding to the new value of resisting bond length $L_{R f i}^{n+1}$ (Fig. 5). The leap of the point $\left(V_{f i} ; L_{R f i} ; \delta_{L i}\right)$ representative of the state of the strip from one bond- 
based constitutive law to the other is only visible in a three dimensional representation, as long as the concrete fracture is superficial (Fig. 5c,e). Where superficial concrete fracture means that, each time the point representative of the state of the strip leaps from one bond-based constitutive law to another, the updated value of $L_{R f i}$ is larger or equal to the necessary bond transfer length $L_{t r}^{b d}\left(\delta_{L i}\right)$ (Fig. 5c,e). On the contrary, the leap of the point $\left(V_{f i} ; L_{R f} ; \delta_{L i}\right)$ representative of the state of the strip from one bond-based constitutive law to the other is also visible in a two dimensional representation, as long as the concrete fracture is deep (Fig. 5a,d). Where deep concrete fracture means that, when the point representative of the state of the strip leaps to the last bond-based constitutive law, the new value of $L_{R f i}$ is smaller than the necessary bond transfer length $L_{t r}^{b d}\left(\delta_{L i}\right)$ corresponding to the current value of $\delta_{L i}$ (Fig. 5a,d). When the point $\left(V_{f i} ; L_{R f i} ; \delta_{L i}\right)$ representative of the state of the strip eventually leaps on the bond-based constitutive law of a strip with null resisting bond length, the ultimate configuration is characterized by a semi-conical concrete fracture whose vertex coincides with the strip free end. It can also happen that, after an initial semi-conical concrete fracture, at a certain point of the loading process, the strip ruptures and the point $\left(V_{f i} ; L_{R f} ; \delta_{L i}\right)$ representative of the state of the strip abruptly falls on the plane $\left(L_{R f i} ; \delta_{L i}\right)$ and the relevant force $V_{f i}$ annuls (Fig. 5c,f).

Note that, whatever the failure mode characterizing the specific single NSM FRP strip, due to the continuity characterizing the surface envelope of the bond-based constitutive laws, it always exists an equivalent value of the resisting bond length $L_{R f i}^{e q}$, which is the value of the resisting bond length to which corresponds a bond-based constitutive law $V_{f i}^{b d}\left(L_{R f i}^{e q} ; \delta_{L i}\right)$ whose peak value $V_{f i}^{b d, \text { max }}$ is equal to the peak value $V_{f i}^{\max }$ of the comprehensive constitutive law $V_{f i}\left(L_{R f i}^{0} ; \delta_{L i}\right)$ of the initial value of the resisting bond length $L_{R f i}^{0}$ (Fig. 5).

Ultimately, the comprehensive constitutive law of a single NSM FRP strip mounted on a concrete prism can be one of the following types (Fig. 4a): (1) either rupture of the strip, preceded or not by a superficial semi-conical fracture, or deep concrete semi-conical fracture up to the free end; (2) deep concrete semi-conical fracture with the concrete fracture stopping midway between loaded and free end; superficial concrete fracture with the last value assumed by $L_{R f i}$ shorter (3), equal (4) or larger (5) than the effective resisting bond length.

In the following of this paragraph, a parametric study, regarding the influence of each of the geometrical-mechanical parameters on the peak value $V_{f i}^{\max }$ of the comprehensive constitutive law $V_{f i}\left(L_{R f i} ; \delta_{L i}\right)$ of a single NSM FRP strip, is presented. Note that even in this case, each parameter was varied within a range comprehending values a little beyond those having a strict physical confirmation, in order to asses not only their influence on the physical behavior of an NSM FRP strip, but also their influence from a mere analytical-numerical standpoint. 
Besides the parameters already introduced in the previous paragraph, the comprehensive behavior of a single NSM FRP strip also depends on (Table 1): strip tensile strength $f_{f u}$, angle between axis and generatrices of the concrete semiconical fracture surface $\alpha$ (Fig. $4 \mathrm{~b}, \mathrm{c}$ ). Among the derived parameters, the concrete average tensile strength $f_{c t m}$, function of $f_{c m}$ is, in the present work, obtained as function of the concrete compressive strength by means of the formulae reported by the CEB Fip Model Code 90.

The curves representing the variation of $V_{f i}^{\max }$ with respect to the FRP strip tensile strength $f_{f u}$ present a bi-linear elastic-perfectly-plastic behavior for whatever value of $L_{R f i}$ (Fig. 6a). Along the first linear branch, the failure mode is rupture of the strip, due to the reduced value of the strip tensile strength $V_{f}^{t r}\left(a_{f} \cdot b_{f} \cdot f_{f u}\right)$. Along the second linear branch, the failure mode is governed by superficial concrete fracture for the shorter values of the initial resisting bond length and by deep concrete fracture for the larger values of the initial resisting bond length. Note that, since the value of $f_{f u}$ does not affect the pure bond-based behavior of a NSM FRP strip, for a given value of $L_{R f i}^{0}$ and for values of $f_{f u}$ larger than the one in correspondence of which the strip no longer ruptures, the ultimate configuration and the relevant peak load $V_{f i}^{\max }$ no longer change by increasing $f_{f u}$. Note also that the curves providing the values of $V_{f i}^{\max }$ as function of $f_{f u}$ for the various values of $L_{R f i}^{0}$ tend to a limit curve which defines the envelope of the various curves. In fact, for the values herein assumed for the other parameters, the curve providing $V_{f i}^{\max }$ as function of $f_{f u}$ is exactly the same regardless of the value assumed by $L_{R f i}^{0}$ for values of $L_{R f i}^{0}$ larger than $400 \mathrm{~mm}$ (Fig. 6a).

The curves $V_{f i}^{\max }\left(E_{f} ; L_{R f i}^{0}\right)$ providing $V_{f i}^{\max }$ as function of the strip Young's Modulus $E_{f}$ present a pseudo bi-linear trend for whatever value of $L_{R f i}^{0}$. Along the first branch, the ultimate behavior is characterized by superficial concrete fracture and, since the equivalent values of the resisting bond length are larger or equal to the corresponding values of the effective resisting bond length ( $L_{R f i}^{e q}\left(E_{f}\right) \geq L_{R f e}\left(E_{f}\right)$ ), the same trend characterizing the pure-bond behavior can be found (Fig. 6d). Nevertheless, due to the reduction of $L_{R f i}^{0}$ ascribed to the superficial concrete fracture, the maximum value of $V_{f i}^{\max }$, for a given value of $L_{R f i}^{0}$, is smaller than the maximum of $V_{f i}^{b d, \text { max }}$ for the same value of $L_{R f i}^{0}$. The pseudo horizontal branches of the curves $V_{f i}^{\max }\left(E_{f} ; L_{R f i}^{0}\right)$ correspond, for the shorter values of $L_{R f i}^{0}(<350 \mathrm{~mm})$, to a deep concrete fracture failure mode and, for the longer values of $L_{R f i}^{0}(\geq 350 \mathrm{~mm})$, to the rupture of the strips. The horizontal branches of the curves $V_{f i}^{\max }\left(E_{f} ; L_{R f i}^{0}\right)$ tend, for increasing values of $L_{R f i}^{0}$, to the limit curve corresponding to 
the value of the strip rupture capacity $V_{f}^{t r}=42.0 \mathrm{kN}$. Note that, on the horizontal branches of the curves $V_{f i}^{\max }\left(E_{f} ; L_{R f i}^{0}\right)$ for the shorter values of $L_{R f i}^{0}$, despite a fluctuation, due to concrete fracture, around an average value, $V_{f i}^{\max }$ is almost constant even if, by increasing $E_{f}$, concrete fracture deepens and $L_{R f i}^{e q}$ progressively becomes a smaller aliquot of $L_{R f i}^{0}$. This is due to the fact that, by increasing $E_{f}$, the values of the resisting bond lengths necessary to transfer the same value of $V_{f i}^{b d, \max }$ reduce (Bianco 2008).

The curves $V_{f i}^{\max }\left(a_{f} ; L_{R f i}^{0}\right)$ and $V_{f i}^{\max }\left(b_{f} ; L_{R f i}^{0}\right)$ providing the value of $V_{f i}^{\max }$ as function of the strip cross section dimensions present, for whatever value of $L_{R f i}^{0}$, a pseudo bi-linear trend (Fig. 6b,e). Along the first branch of those curves, due to the reduced area of the strip cross section, the failure mode is rupture of the strip and $V_{f i}^{\max }$ increases linearly for increasing values of either $a_{f}$ or $b_{f}$. Along the second branch of those curves, the ultimate configuration is of the mixed-semi-cone-plus-debonding type and, for the shorter values of $L_{R f i}^{0}$, the failure mode is superficial concrete fracture while for the longer values of $L_{R f i}^{0}$, the failure mode is deep concrete fracture. The horizontal branches of those curves tend, for increasing values of $L_{R f i}^{0}$, to the limit curve corresponding to the value of approximately $V_{f i}^{\max }=62.0 \mathrm{kN}$. From a certain value of $L_{R f i}^{0}$ on $\left(L_{R f i}^{0} \geq 350 \mathrm{~mm}\right.$ for $a_{f}$ and $L_{R f i}^{0} \geq 400 \mathrm{~mm}$ for $\left.b_{f}\right)$, the curves above overlap on the limit curve and the maximum value of $V_{f i}^{\max }$ does not further increase for increasing values of $L_{R f i}^{0}$.

The curves $V_{f i}^{\max }\left(a_{c} ; L_{R f i}^{0}\right)$ and $V_{f i}^{\max }\left(b_{c} ; L_{R f i}^{0}\right)$ providing the value of $V_{f i}^{\max }$ as function of the concrete prism cross section dimensions present, for whatever value of $L_{R f i}^{0}$, a pseudo bi-linear trend (Fig. 6c,f). Along the first branch of those curves, since the prism cross section is small, the successive co-axial semi-conical fracture surfaces, whose axis is a progressively larger amount of $L_{R f i}^{0}$, soon intersect the concrete prism faces (Fig. $\left.4 \mathrm{~b}, \mathrm{c}\right)$. Thus, the concrete semiconical fracture capacity $V_{f i}^{c f}$, obtained integrating the concrete average tensile strength throughout the surface resulting from the intersection of the right semi-conical surface with the prism faces, is very low (Bianco et al. 2009a-b). Due to the small value of the concrete fracture capacity, along the first branch of the curves $V_{f i}^{\max }\left(a_{c} ; L_{R f i}^{0}\right)$ and $V_{f i}^{\max }\left(b_{c} ; L_{R f i}^{0}\right)$, the failure mode is very deep concrete fracture with the vertex of the last semi-conical fracture surface reaching the strip free end (Fig. 4b,c). Along the second branch of those curves, for increasing values of either $a_{c}$ or $b_{c}$, and for the shorter values of $L_{R f i}^{0}$, concrete fracture becomes, as long as the largest semi-conical surface intersects the prism walls, progressively more superficial. From the value of either $a_{c}$ or $b_{c}$, in correspondence of which the largest semi-conical 
surface no longer intersects the prism faces, the ultimate configuration and the corresponding value of $V_{f i}^{\max }$, for a given value of $L_{R f i}^{0}$, remains practically unchanged. Along the second branch of those curves, for values of $L_{R f i}^{0}$ larger than a certain value ( $L_{R f i}^{0} \geq 300 \mathrm{~mm}$ for $a_{c}$ and $L_{R f i}^{0} \geq 250 \mathrm{~mm}$ for $b_{c}$ ), the failure mode is governed by the strip rupture and those branches overlap on the limit curve corresponding to the value of $V_{f i}^{\max }=V_{f}^{t r}=42.0 \mathrm{kN}$ (Fig. $\left.6 \mathrm{c}, \mathrm{f}\right)$.

The curve $V_{f i}^{\max }\left(L_{R f i}^{0}\right)$ providing the value of $V_{f i}^{\max }$ as function of the value of $L_{R f i}^{0}$ also presents a pseudo bi-linear trend (Fig. 7c). Along the first branch, the failure mode is concrete semi-conical fracture, either superficial or deep, with an ultimate configuration of the mixed-semi-cone-plus-debonding type. Along the second branch, the failure mode is rupture of the strip. Note that even in this case as for the pure bond behavior, it is possible to single out an effective value value of $L_{R f i}^{0}$, which can be labeled as comprehensive effective resisting bond length $L_{R f e}^{0}$, beyond which any further increase of $L_{R f i}^{0}$ does not produce any further gain in terms of $V_{f i}^{\max }$. In general, the comprehensive effective resisting bond length $L_{R f e}^{0}$, due to the occurrence of one of either concrete fracture or strip rupture, is shorter than the effective resisting bond length $L_{R f e}$ (see also Fig. ).

The curves $V_{f i}^{\max }\left(\alpha ; L_{R f i}^{0}\right)$ and $V_{f i}^{\max }\left(f_{c m} ; L_{R f i}^{0}\right)$ providing the value of $V_{f i}^{\max }$ as function of either of the parameters defining the concrete mechanical properties, either $f_{c m}$ or $\alpha$, present, for whatever value of $L_{R f i}^{0}$, a nonlinear trend in which, in the most general case, three successive branches can be singled out (Fig. 7a,b). Along the first branch, for small values of either $f_{c m}$ or $\alpha$, since concrete mechanical properties are very low, concrete fracture is so deep to reach the strip free extremity. This first branch of the curve is continuous since, going from one value to the other of the parameter analyzed ( $f_{c m}$ or $\alpha$ ), the peak value $V_{f i}^{\max }$ is always equal to the concrete semi-conical fracture capacity $V_{f i}^{c f}$ associated to the maximum semi-conical surface that can form inside the concrete prism. Along this first branch, the concrete fracture capacity $V_{f i}^{c f}\left(L_{R f i}^{0}\right)$, integral of $f_{c t m}$ on the maximum semi-conical surface varies with continuity for an increase of either $f_{c t m}\left(f_{c m}\right)$ or $\alpha$. In this latter case, $V_{f i}^{c f}\left(L_{R f i}^{0}\right)$ varies with continuity if either the maximum semi-conical surface intersects (for larger values of $\alpha$ ) or not (for smaller values of $\alpha$ ) the concrete prism faces (Fig. 4). By increasing the values of either $f_{c m}$ or $\alpha$, concrete fracture progressively becomes more superficial and the equivalent value of the resisting bond length becomes a progressively larger aliquot of $L_{R f i}^{0}$. Along the second branch of the curves $V_{f i}^{\max }\left(\alpha ; L_{R f i}^{0}\right)$ and $V_{f i}^{\max }\left(f_{c m} ; L_{R f i}^{0}\right)$ the ultimate configuration is of the mixed-semi-cone-plus-debonding 
type. Such second branch is characterized by a two-fold fluctuation of the values $V_{f i}^{\max }$, by varying either of the parameters analyzed $\left(f_{c m}\right.$ or $\alpha$ ) and for a given value of $L_{R f i}^{0}$, around the values corresponding to an average and continuous curve: a micro-fluctuation ad a macro-fluctuation. The former is a fluctuation that has a numerical origin and is due to the way in which the concrete semi-conical fracture phenomenon was modeled (Bianco 2008). The latter, which consists in the presence of some steps upwards, is due to the fact that, for increasing values of the concrete mechanical properties, the number of successive reductions of $L_{R f i}^{0}$ (Fig. 4), contextual to the occurrence of successive concrete semi-conical fractures, reduces for increasing values of either of the parameters analyzed $\left(f_{c m}\right.$ or $\left.\alpha\right)$. Along the third branch, attained indeed only for the larger values of $L_{R f i}^{0}$ and for increasing values of the concrete mechanical properties, the ultimate configuration is composed of a superficial semi-conical concrete fracture followed by the rupture of the strip itself. Such third branch is therefore horizontal and equal to the value of the strip rupture capacity that, for the values herein assumed for the other parameters, is equal to $42.0 \mathrm{kN}$. The curves $V_{f i}^{\max }\left(\alpha ; L_{R f i}^{0}\right)$ and $V_{f i}^{\max }\left(f_{c m} ; L_{R f i}^{0}\right)$, for increasing values of $L_{R f i}^{0}$, progressively become closer to each other up to overlapping on a limit curve for values of $L_{R f i}^{0}$ larger than a certain limit value that, for the values herein assumed for the other parameters, is equal to $450 \mathrm{~mm}$ for $\alpha$ and to $300 \mathrm{~mm}$ for $f_{c m}$. Note that the curves $V_{f i}^{\max }\left(f_{c m} ; L_{R f i}^{0}\right)$ start from a value of $f_{c m}$ equal to 8.0 MPa. This is due to the fact that the concrete average tensile strength $f_{c t m}$ was calculated from the concrete average compressive strength by means of the formulae reported by the CEB-FIP Model Code 90 that provide values of $f_{c t m}$ larger than zero for values of $f_{c m}$ larger than $8.0 \mathrm{MPa}$. Note also that, if either $f_{c m}$ or $\alpha$ were increased infinitely, for a given value of $L_{R f i}^{0}$, the value of $V_{f i}^{\max }$ would tend to the corresponding bond-based peak load $V_{f i}^{b d, \text { max }}\left(L_{R f i}^{0}\right)$, as long as this latter is smaller than the strip rupture capacity.

The peak force $V_{f i}^{\max }$ that an NSM FRP strip of a given resisting bond length $L_{R f i}^{0}$ can resist does not vary by increasing the value of the parameter $\tau_{0}$, characterizing the adopted local bond stress-slip relationship, for whatever value of the initial resisting bond length $L_{R f i}^{0}$ (Fig. 8a). Actually, the parameter $\tau_{0}$ was already found not to affect the peak bondtransferred force $V_{f i}^{b d, \max }$ (Fig. 3a). Nonetheless, due to the occurrence of other phenomena such as either concrete tensile fracture or strip rupture, the peak value of the curve $V_{f i}^{\max }\left(\tau_{0} ; L_{R f i}^{0}\right)$ is lower than the peak value of the corresponding curve $V_{f i}^{b d, \max }\left(\tau_{0} ; L_{R f i}\right)$ for a value of $L_{R f i}=L_{R f i}^{0}$. For the shorter values of $L_{R f i}^{0}$, the failure mode is 
governed by concrete tensile fracture, either superficial or deep while, for values of $L_{R f i}^{0}$ larger or equal to a certain value, the failure mode is the strip rupture.

The values of the parameters $\tau_{1}$ and $\tau_{2}$ have a negligible influence on the peak value of the comprehensive constitutive law of a NSM FRP strip of a given resisting bond length $L_{R f i}^{0}$ (Fig. 8b,c). In fact, the curves $V_{f i}^{\max }\left(\tau_{1} ; L_{R f i}^{0}\right)$ and $V_{f i}^{\max }\left(\tau_{2} ; L_{R f i}^{0}\right)$ do not show appreciable variations, by increasing either $\tau_{1}$ or $\tau_{2}$, for whatever value of $L_{R f i}^{0}$. For the shorter values of $L_{R f i}^{0}$, the failure mode is governed by concrete tensile fracture, either superficial or deep while, for values of $L_{R f i}^{0}$ larger or equal to a certain value, the failure mode is the strip rupture.

Each of the bond slip values characterizing the local bond stress-slip relationship, either $\delta_{1}$ or $\delta_{2}$ or $\delta_{3}$, has a marginal influence on the peak value of the comprehensive constitutive law of a NSM FRP strip of a given value of the initial resisting bond length $L_{R f i}^{0}$ (Fig. 8d-f). For the shorter values of $L_{R f i}^{0}$, the failure mode is governed by concrete tensile fracture, either superficial or deep while, for values of $L_{R f i}^{0}$ larger or equal to a certain value, the failure mode is the strip rupture. Note that the curves providing $V_{f i}^{\max }$ as function of either of the various parameters defining the local bond stress-slip relationship tend, for increasing values of $L_{R f i}^{0}$, to overlap to the limit curve corresponding to the strip rupture $V_{f i}^{\max }=V_{f}^{t r}$

\section{NSM FRP strips' shear strength contribution to a RC beam}

The mechanical model adopted in the previous paragraphs, when applied faithfully to its original features (Bianco 2008, Bianco et al. 2009a), allows the shear strength contribution provided by a system of NSM FRP strips to a RC beam $V_{f}$ to be evaluated as function of the CDC opening angle $\gamma$ (Fig. 9). The relation $V_{f}(\gamma)$ may be evaluated for whatever relative geometrical position that the occurred CDC should assume with respect to the system of NSM FRP strips. Nonetheless, in the present work, three geometrical configurations only $(k=1,2,3)$ were taken into consideration: $(k=1)$ the minimum number of strips that can effectively cross the CDC with the first one placed at a distance equal to the strips' spacing $s_{f}$ from the CDC origin; $(k=2)$ an even number of strips symmetrically placed with respect to the crack axis; $(k=3)$ an odd number of strips with the central one attaining the maximum available bond length by being located along the crack axis (Bianco 2008). To evaluate the relationship $V_{f, k}(\gamma)$ it is necessary to introduce, besides the input parameters already introduced in the previous paragraphs, also the following ones (Figure 9): the beam web cross section depth $h_{w}$ and width $b_{w}$, the CDC inclination angle $\theta$ with respect to the beam longitudinal axis, the strips 
inclination angle with respect to the beam longitudinal axis $\beta$ and the CDC opening angle increment $\dot{\gamma}$. The typical relationship $V_{f, k}(\gamma)$, sum of the contribution of the strips effectively crossing the CDC, is characterized by abrupt decays of $V_{f}$ that correspond to the failure of the various strips (Fig. 9c-f). Typically, according to that model, during the loading process of a RC beam strengthened in shear, co-axial semi-conical concrete fractures form around each of the strips effectively crossing the CDC since the first load steps. Due to the formation of successive co-axial semiconical fracture surfaces around each of the strips, the resulting concrete fracture envelope surface, starting from the CDC, progressively penetrates within the web core on either side of the CDC plane (Bianco et al. 2009a).

The mechanical model mentioned above, was applied to evaluate the influence of each of the input parameters (Table 2) on the peak NSM FRP strips shear strength contribution to a RC beam $V_{f, k}^{\max }$ and the obtained results are presented below.

The Reference Beam (RB) assumed for the parametric studies along with the range of values assumed for each parameter, are listed in Table 2. The values of the parameters characterizing the RB have been chosen in such a way to result approximately the average values of those that can be met in real practice. In some cases, the specific parameter is varied within a range that comprehends values a little beyond those having a strict physical confirmation, in order to assess not only their influence on the physical behavior of RC beams strengthened in shear by the NSM technique, but also their influence from a mere analytical-numerical standpoint. The range of variation of $f_{c m}$ was limited to the values 10-90 MPa (Table 2) for which concrete can be considered as structural concrete, in accordance to the international regulations (e.g. CEB-FIP Model Code 1990).

The load step $\dot{\gamma}$ slightly influences, for the range of values in which it was herein varied $\left(0.0001^{\circ}-0.01^{\circ}\right)$, the peak NSM shear strength contribution $V_{f, k}^{\max }$ (Fig. 10a). In this scenario, it is deemed reasonable to assume a value of the load step equal to $\dot{\gamma}=0.01^{\circ}$ since it guarantees a good compromise between accuracy of prediction and computational demand.

The peak NSM shear strength contribution $V_{f, k}^{\max }$ decreases by assuming increasing values of the CDC inclination angle $\theta$ since, other parameters being the same, the number of strips effectively crossing the CDC decreases (Fig. 10b).

The peak NSM shear strength contribution $V_{f, k}^{\max }$, for the range of values herein assumed for the other parameters, increases linearly for increasing value of the web cross section depth $h_{w}$ (Fig. 10c), since both the number of strips effectively crossing the CDC and their available bond lengths increase in turn. Actually, up to a certain value of $h_{w}$, the linear increase of $V_{f, k}^{\max }$ is primarily due to the increase of the strips available bond lengths while, for larger values of 
$h_{w}$, since the strips available bond lengths start to be larger than the comprehensive effective resisting bond length (Fig. 7c), the linear increase of $V_{f, k}^{\max }$ is due to the increase in the number of strips effectively crossing the CDC.

By increasing the RC beam web cross section width $b_{w}$ (Fig. 10d), the peak NSM shear strength contribution $V_{f, k}^{\max }$ presents a two-phases behavior with an almost clear-cut division between themselves in correspondence of a value of approximately $b_{w}=15 \mathrm{~cm}$. For values of the web width $b_{w} \leq 15 \mathrm{~cm}$, by increasing $b_{w}, V_{f, k}^{\max }$ increases almost linearly up to a maximum while, for values of the web width $b_{w}>15 \mathrm{~cm}$, any further increase of $b_{w}$ does not yield any further increase of $V_{f, k}^{\max }$. This is due to the fact that, for $b_{w} \leq 15 \mathrm{~cm}$, the strips symmetrically placed on the opposite sides of the strengthened web interact transversally with each other while, for values of $b_{w}>15 \mathrm{~cm}$, due to the lack of interaction between strips placed on the opposite sides of the beam web, any further increase of $b_{w}$ does not produce any change in the overall response.

The peak NSM shear strength contribution $V_{f, k}^{\max }$ presents, with respect to either of the CFRP strip's cross-section dimensions ( $a_{f}$ or $b_{f}$ ), a three-phases trend (Fig. 10e-f). In the first phase ( $a_{f} \leq 1.5 \mathrm{~mm}$ or $b_{f} \leq 8 \mathrm{~mm}$ ), the prevalent failure mode is tensile rupture of the strips and $V_{f, k}^{\max }$ increases almost linearly by increasing either $a_{f}$ or $b_{f}$. In fact, after a soon and shallow concrete fracture, most of the strips crossing the CDC rupture due to their reduced crosssection area. In the intermediate stretch $\left(1.5<a_{f} \leq 4.5 \mathrm{~mm}\right.$ or $\left.8<b_{f} \leq 12 \mathrm{~mm}\right) V_{f, k}^{\max }$ increases, by increasing either $a_{f}$ or $b_{f}$, by a decreasing rate since the number of strips undergoing rupture progressively reduces and the number of strips failing for concrete fracture, either superficial or deep and up to the free end, increases. Along the third stretch ( $a_{f}>4.5 \mathrm{~mm}$ or $b_{f}>12 \mathrm{~mm}$ ), all of the strips effectively crossing the CDC fail for either deep or superficial concrete fracture and their contribution $V_{f i}^{\max }$ to the overall shear strength contribution $V_{f, k}^{\max }$ no longer depends on either $a_{f}$ or $b_{f}$ (Fig. 6). However, for very large values of either of the strip cross-section dimensions, the model progressively loses accuracy underestimating the concrete fracture capacity since the approximation of the concrete fracture surface as a semi-cone whose axis lies on the web face (Fig. 4) may no longer be acceptable (Bianco et al. 2009a).

By increasing the spacing $s_{f}$ between adjacent strips, even though the contribution provided by the single strip $V_{f i}^{\max }$ would increase as a consequence of the increase of the cross section of the relevant concrete prism (Figs. 9a and 4b-c), the overall contribution to the peak NSM shear strength $V_{f, k}^{\max }$ significantly decreases (Fig. 11a) since the number of strips effectively crossing the CDC decreases in turn. For the third geometrical configuration $(k=3)$, for which, from a 
certain value of $s_{f}$ on, the number of strips effectively crossing the CDC remains unchanged and equal to one central strip only, the same plastic stretch, already highlighted for the single strip (Fig. 6c) can be found again.

By increasing the value of the inclination of the strips with respect to the beam axis $\beta$, the peak NSM shear strength contribution decreases (Fig. 11b) mainly because, other parameters being the same, both the number of strips effectively crossing the CDC and their initial available bond lengths decrease.

The strip's Young's Modulus $E_{f}$ has, on the whole and for the values assumed in the present study ( $E_{f}=100 \div 250 G P a$ ), a negligible influence on $V_{f, k}^{\max }$ (Fig. 11c). In fact, for the range of values of $E_{f}$ analyzed, already the single strip peak contribution $V_{f i}^{\max }$ does not vary for increasing values of $E_{f}$, for each value of $L_{R f i}^{0}$ and whatever the ultimate configuration (Fig. 6d). Thus, even the overall shear strength contribution $V_{f, k}^{\max }$ does not vary for increasing values of $E_{f}$, within that range.

The trend of the peak NSM shear strength contribution $V_{f, k}^{\max }$ with respect to the strip tensile strength $f_{f u}$ shows two phases separated by an almost clear-cut turning point approximately in correspondence of the value of $3.0 \mathrm{GPa}$ (Fig. 11d). For values of $f_{f u}$ smaller than $3.0 \mathrm{GPa}$, the ultimate behavior is governed by the prevalent rupture of the strips. For values of $f_{f u}$ equal to or larger than $3.0 \mathrm{GPa}$, the ultimate behavior is governed by concrete fracture and, whatever the depth of concrete fracture penetration, each strip's peak contribution does not change for increasing values of $f_{f u}$, whatever their $L_{R f i}^{0}$ (Fig. 6a).

The variation of $V_{f, k}^{\max }$ with respect to the concrete mechanical properties, either $f_{c m}$ or $\alpha$, presents a non-linear trend in which three successive stretches can be singled out (Fig. 11e-f). For values of $\alpha \leq 30^{\circ}$ and $f_{c m} \leq 30 M P a$, the prevailing failure mode of most of the strips effectively crossing the CDC is concrete tensile fracture reaching the strips' free ends (Fig. 7a-b). Along the second stretch, for values of $30^{\circ}<\alpha \leq 40^{\circ}$ and $30<f_{c m} \leq 60 M P a$, by progressively increasing the concrete mechanical properties, either $f_{c m}$ or $\alpha$, concrete fracture progressively becomes shallower (Fig. 4c) and $V_{f, k}^{\max }$ increases by a decreasing rate. In fact, along the second stretch, since the curve $V_{f i}^{\max }(\alpha)$ or $V_{f i}^{\max }\left(f_{c m}\right)$ are characterized by a $L_{R f i}^{0}$-dependent rate (Fig. $7 \mathrm{a}-\mathrm{b}$ ), and $V_{f, k}^{\max }$ is equal to the sum of the contribution $V_{f i}^{\max }$ of strips with different values of $L_{R f i}^{0}, V_{f, k}^{\max }$ increases by a decreasing rate. Along the third stretch, for values of $\alpha>40^{\circ}$ and $f_{c m}>60 \mathrm{MPa}, V_{f, k}^{\max }$ does not further vary for increasing values of either $f_{c m}$ or $\alpha$. In fact, for values of 
$\alpha>40^{\circ}$ and $f_{c m}>60 M P a$, the peak of the single strips' contribution $V_{f i}^{\max }$ does not vary, independently of the failure mode (Fig. 7a-b).

As already found out for a single NSM FRP strip subjected to an increasing imposed end slip (Fig. 8), the values of the parameters defining the local bond stress-slip relationship do not affect, for the values of the other parameters herein assumed, the peak NSM FRP strips shear strength contribution (Fig. 12). In fact, due to the occurrence of other phenomena such as either concrete semi-conical tensile fracture or strip rupture, the peak bond-transferred force $V_{f i}^{b d, \text { max }}$ that the initial values of the resisting bond length would be capable of transferring, can never be attained.

\section{Conclusions}

A mechanical model, recently developed to evaluate the NSM FRP strips shear strength contribution to a RC beam throughout its loading process, was herein applied to carry out parametric studies. These latter encompass the studies of the influence of each geometrical-mechanical parameter on: 1) the peak load that a single NSM FRP strip can transfer, through bond stresses, to the surrounding concrete, neglecting both concrete semi-conical fracture and strip rupture; 2) the peak load that a single NSM FRP strip can comprehensively transfer, taking also into account the possible occurrence of either concrete fracture or strip rupture; 3) the peak shear strength contribution that a group of NSM FRP strips can provide to a $\mathrm{RC}$ beam.

The bond-based constitutive law of a single NSM FRP strip, which is the curve providing the force that a strip with a given value of resisting bond length can transfer, through bond stresses, as function of the imposed end slip, forms a continuous surface when plotted in a three-dimensional graph for increasing values of resisting bond length. The peak bond-transferred force, which is the peak value of the strip's constitutive law, increases, for increasing values of the resisting bond length, only up to a certain threshold, labeled effective resisting bond length, beyond which any further length increase does not produce any further force gain. The peak bond-transferred force increases, with a rate that is as larger as larger is the value of the resisting bond length, for increasing values of either the strip's Young's Modulus or the strip's cross section dimensions. The peak bond-transferred force increases also, with a rate that is as larger as larger is the value of the resisting bond length, for increasing values of most of the parameters defining the local bond-stress slip relationship.

The comprehensive behavior of a single NSM FRP strip subjected to an increasing imposed end slip is characterized by the formation of successive co-axial semi-conical concrete fractures which can stop progressing midway between loaded and free end, maybe followed by strip rupture if the strip rupture capacity is attained, or progress up to the free end. Contextually at the occurrence of each concrete fracture, the initial value of the resisting bond length progressively 
reduces and the point describing the strip's comprehensive constitutive law in a three dimensional representation leaps from one bond-based constitutive law to another.

If either the concrete fracture reaches the strip's free end or the strip ruptures, the strip's comprehensive constitutive law abruptly annuls. Since the bond-based constitutive law of an NSM FRP strip forms a continuous surface for increasing values of the resisting bond length, it is always possible to single out an equivalent value of the initial resisting bond length whose bond-based constitutive law has a peak value equal to the peak load of the comprehensive constitutive law of the initial resisting bond length.

The dependence of the peak value of the comprehensive constitutive law on most of the input parameters shows a nonlinear, initial resisting-bond-length-value-dependent and pseudo elasto-plastic trend. The non-linearity is due to the fact that, for a given value of initial resisting bond length, for increasing values of the studied parameter, both the failure mode and the ultimate configuration attained by the strip progressively change.

The peak value of the comprehensive constitutive law increases by increasing the initial value of the resisting bond length up to a certain value, labeled effective value of the initial resisting bond length, beyond which any further length increase does not yield any further force gain.

The influence of each one of the parameters defining the local bond stress-slip relationship on the peak value of the comprehensive constitutive law of a single NSM FRP strip with a given value of initial resisting bond length is negligible. In fact, the premature occurrence of either concrete semi-conical fracture or strip rupture does not allow to attain the peak bond-transferred force that a single strip, with the given value of initial resisting bond length, would be capable to attain.

The behavior of a system of NSM FRP strips contributing to the shear strength of a RC beam is extremely complex since the strips effectively contributing to the beam shear strength, intersecting the CDC plane, are: 1) characterized by different values of the initial resisting bond length, 2) not necessarily orthogonal to the CDC, 3) subjected to different values of imposed end slip and 4) bi-directionally interact between each other.

The curve providing the NSM FRP strips shear strength contribution as function of the CDC opening angle is characterized by abrupt force reductions due to the strips' failure, whatever the failure mode they singly undergo. The dependence of the peak NSM shear strength contribution on most of the input geometrical-mechanical parameters is extremely non-linear.

As already outlined for the comprehensive behavior of a single NSM FRP strip, a variation of each of the parameters defining the local bond stress-slip relationship yields a negligible variation of the peak NSM shear strength contribution, due to the premature occurrence of either concrete semi-conical fracture or strip rupture.

\section{Acknowledgements}


The authors of the present work wish to acknowledge the support provided by the "Empreiteiros Casais", $\mathrm{S} \& \mathrm{P} \otimes$, degussa $^{\circledR}$ Portugal, and Secil (Unibetão, Braga). The study reported in this paper forms a part of the research program “CUTINEMO” supported by FCT, PTDC/ECM/73099/2006. Also, this work was carried out under the auspices of the Italian DPC-ReLuis Project (repertory n. 540), Research Line 8, whose financial support is greatly appreciated. 


\section{References}

Bianco, V., Barros, J.A.O., Monti, G., (2006). "Shear Strengthening of RC beams by means of NSM laminates: experimental evidence and predictive models", Technical report 06-DEC/E-18, Dep. Civil Eng., School Eng. University of Minho, Guimarães- Portugal.

Bianco, V., Barros, J.A.O., Monti, G., (2007). "Shear Strengthening of RC beams by means of NSM strips: a proposal for modeling debonding", Technical report 07-DEC/E-29, Dep. Civil Eng., School Eng. University of Minho, Guimarães- Portugal.

Bianco, V., (2008). "Shear Strengthening of RC beams by means of NSM FRP strips: experimental evidence and analytical modeling", PhD Thesis, Dept. of Structural Engrg. and Geotechnics, Sapienza University of Rome, Italy, submitted on December 2008.

Bianco, V., Barros, J.A.O., Monti, G., (2009a). "Three dimensional mechanical model for simulating the NSM FRP strips shear strength contribution to RC beams", Engineering Structures, 31(4), April 2009, 815-826.

Bianco, V., Barros, J.A.O., Monti, G., (2009b). "Bond Model of NSM FRP strips in the context of the Shear Strengthening of RC beams”, ASCE Journal of Structural Engineering, 135(6), June 2009.

CEB-FIP Model Code 90, (1993) Bulletin d'Information $N^{\circ} 213 / 214$, Final version printed by Th. Telford, London, (1993; ISBN 0-7277-1696-4; 460 pages).

Dias, S.J.E.; Barros, J.A.O., "Shear strengthening of RC T-section beams with low concrete using NSM CFRP laminates", Cement and Concrete Composites Journal, doi:10.1016/j.cemconcomp.2010.10.002, 2011 b.

Dias, S.J.E.; Barros, J.A.O., "Experimental behaviour of RC beams shear strengthened with NSM CFRP laminates", Strain International Journal, doi: 10.1111/j.1475-1305.2010.00801.x, 2011a.

Dias, S.J.E., Bianco, V., Barros, J.A.O., Monti, G., (2007). "Low strength concrete T cross section RC beams strengthened in shear by NSM technique", Workshop-Materiali ed Approcci Innovativi per il Progetto in Zona Sismica e la Mitigazione della Vulnerabilità delle Strutture, University of Salerno, Italy, 12-13 February.

Dias, S.J.E. and Barros, J.A.O., (2008). "Shear Strengthening of T Cross Section Reinforced Concrete Beams by Near Surface Mounted Technique”, Journal of Composites for Construction, ASCE, Vol. 12, No. 3, pp. 300-311.

Mohammed Ali, M.S., Oehlers, D.J., Seracino, R. (2006). "Vertical shear interaction model between external FRP transverse plates and internal stirrups", Engineering Structures 28, 381-389.

Mohammed Ali, M.S., Oehlers, D.J., Griffith, M.C., Seracino, R. (2007). "Interfacial stress transfer of near surface-mounted FRP-to-concrete joints”, Engineering Structures 30, 1861-1868.

Rizzo, A. and De Lorenzis, L., (2009) "Behaviour and capacity of Rc beams strengthened in shear with NSM FRP reinforcement", Construction and Building Materials, Vol. 3, n. 4, April 2009, 1555-1567. 
Sena-Cruz, J.M. (2004). "Strengthening of concrete structures with near-surface mounted CFRP laminate strips" PhD Thesis, Department of Civil Engineering, University of Minho, Guimarães-Portugal.

Sena-Cruz, J.M., Barros, J.A.O., (2004). "Bond between near-surface mounted CFRP laminate strips and concrete", Journal of Composites for Construction, ASCE, Vol. 8, No. 6, pp. 519-527.

Yuan, H., Teng, J.G., Seracino, R., Wu, Z.S., Yao, J. (2004). "Full-range behavior of FRP-to-concrete bonded joints", Engineering Structures, 26, 553-565. 


\section{TABLE CAPTIONS}

Table 1 - Values for the input parameters adopted for the parametric studies regarding the behavior of a single NSM FRP strip.

Table 2-Values for the input parameters adopted for the parametric studies regarding the peak shear strength contribution provided by a system of NSM FRP strips to a RC beam.

Table 1 - Values for the input parameters adopted for the parametric studies regarding the behavior of a single NSM FRP strip.

\begin{tabular}{|c|c|c|c|c|c|c|c|c|c|c|c|c|c|c|c|}
\hline & $E_{f}$ & $f_{f u}$ & $f_{c m}$ & $\alpha$ & $a_{f}$ & $b_{f}$ & $a_{c}$ & $b_{c}$ & $L_{R f i}$ & $\tau_{0}$ & $\tau_{1}$ & $\tau_{2}$ & $\delta_{1}$ & $\delta_{2}$ & $\delta_{3}$ \\
& $G P a$ & $G P a$ & $M P a$ & $\circ$ & $m m$ & $m m$ & $c m$ & $c m$ & $c m$ & $M P a$ & $M P a$ & $M P a$ & $m m$ & $m m$ \\
\hline \hline Reference Strip & 150 & 3.0 & 35.0 & 28.5 & 1.4 & 10.0 & 15.0 & 15.0 & 4.0 & 2.0 & 20.1 & 9.0 & 0.07 & 0.83 & 14.1 \\
\hline Range of variation & $0-300$ & $0-5.0$ & $20-70$ & $0-50$ & $0.0-7.0$ & $0-35$ & $2-40$ & $2-40$ & $0-150$ & $0.5-10.0$ & $9.0-50.0$ & $3.0-19.0$ & $0.05-0.8$ & $0.2-13.0$ & $2.0-25.0$ \\
\hline
\end{tabular}

Table 2 - Values for the input parameters adopted for the parametric studies regarding the peak shear strength contribution provided by a system of NSM FRP strips to a RC beam.

\begin{tabular}{|c|c||c||c|c||c|c|c|c|c|c||c|c||c|c|c|c|c|c|}
\hline & $\begin{array}{c}\dot{\gamma} \\
\circ\end{array}$ & $\begin{array}{c}\theta \\
\circ\end{array}$ & $\begin{array}{c}h_{w} \\
\mathrm{~cm}\end{array}$ & $\begin{array}{c}b_{w} \\
\mathrm{~cm}\end{array}$ & $\begin{array}{c}a_{f} \\
m m\end{array}$ & $\begin{array}{c}b_{f} \\
m m\end{array}$ & $\begin{array}{c}s_{f} \\
\mathrm{~cm}\end{array}$ & $\begin{array}{c}\beta \\
\circ\end{array}$ & $\begin{array}{c}E_{f} \\
G P a\end{array}$ & $\begin{array}{c}f_{f u} \\
G P a\end{array}$ & $\begin{array}{c}f_{c m} \\
M P a\end{array}$ & $\begin{array}{c}\alpha \\
\circ\end{array}$ & $\begin{array}{c}\tau_{0} \\
M P a\end{array}$ & $\begin{array}{c}\tau_{1} \\
M P a\end{array}$ & $\begin{array}{c}\tau_{2} \\
M P a\end{array}$ & $\begin{array}{c}\delta_{1} \\
m m\end{array}$ & $\begin{array}{c}\delta_{2} \\
m m\end{array}$ & $\begin{array}{c}\delta_{3} \\
m m\end{array}$ \\
\hline $\begin{array}{c}\text { Reference } \\
\text { Beam }\end{array}$ & 0.01 & 45.0 & 50.0 & 20.0 & 1.4 & 10.0 & 20.0 & 45.0 & 150.0 & 3.0 & 35.0 & 28.5 & 2.0 & 20.1 & 9.0 & 0.07 & 0.83 & 14.1 \\
\hline $\begin{array}{c}\text { Range of } \\
\text { Variation }\end{array}$ & $1-10^{-4}$ & $20-50$ & $20-70$ & $5-40$ & $1.0-5.5$ & $5-35$ & $5-80$ & $45-90$ & $100-250$ & $1.0-5$ & $10-90$ & $10-45$ & $0.5-5$ & $9.5-35$ & $3.0-19$ & $0.05-0.8$ & $0.2-10$ & $2.0-20$ \\
\hline
\end{tabular}




\section{FIGURE CAPTIONS}

Fig. 1. Bond-based behavior of a single Near Surface Mounted (NSM) FRP strip on a concrete prism: a-b) constitutive law $V_{f i}^{b d}\left(L_{R f i} ; \delta_{l i}\right)$ both in a bi-dimensional and in a three dimensional representation; c) dependence of the maximum bond-transferred force $V_{f i}^{b d, m a x}$ on the resisting bond length $L_{R f i}$; d) concrete prism with a single NSM FRP strip and e) adopted local bond stress-slip relationship.

Fig. 2. Variation of the peak bond-transferred force by a single NSM FRP strip as function of: a) concrete Young's Modulus $E_{c}$, b) strip thickness $a_{f}$, c) concrete prism thickness $a_{c}$, d) FRP Young's Modulus $E_{f}$, e) strip width and f) concrete prism width.

Fig. 3. Influence of the parameters defining the local bond stress-slip relationship on the peak force $V_{f i}^{b d, \text { max }}$ that a single NSM FRP strip can transfer, through bond stresses, to the surrounding concrete.

Fig. 4. Comprehensive behavior of a single NSM FRP strip: a) possible comprehensive constitutive law types, b) successive and co-axial semi-conical fracture surfaces occurring around the NSM strip and c) progressive reduction of the resisting bond length and penetration of the semi-conical fracture within the concrete prism.

Fig. 5. Comprehensive constitutive law $V_{f i}\left(L_{R f i} ; \delta_{L i}\right)$ of a single NSM FRP strip: a,d) deep concrete fracture, b,e) superficial concrete fracture and c,f) strip rupture after a superficial concrete fracture.

Fig. 6. Variation of the peak force that a single strip can comprehensively transfer to the surrounding concrete $V_{f i}^{\max }\left(L_{R f i}^{0}\right)$, as function of: FRP's a) tensile strength $f_{f u}$ and d) Young's Modulus $E_{f}$, strip cross section b) thickness $E_{f}$ and e) width $E_{f}$ and concrete prism cross section c) thickness $a_{c}$ and f) width $b_{c}$.

Fig. 7. Variation of the peak force that a single strip can comprehensively transfer to the surrounding concrete $V_{f i}^{\max }\left(L_{R f i}^{0}\right)$, as function of: a) the angle between axis and generatrices of the semi-conical concrete fracture surface $\alpha$, b) concrete average compressive strength $f_{c m}$ and c) initial resisting bond length $L_{R f i}^{0}$.

Fig. 8. Variation of the peak force that a single strip can comprehensively transfer to the surrounding concrete $V_{f i}^{\max }\left(L_{R f i}^{0}\right)$, as function of the parameters defining the adopted local bond stress-slip relationship.

Fig. 9. NSM FRP strips shear strength contribution to a RC beam: (a-b) schematic representation of the beam web shear-strengthened by NSM FRP strips, (c-d) NSM shear strength contribution to the adopted Reference Beam for two values of the CDC inclination angle and (e-f) for two values of the beam web depth.

Fig. 10. Influence on the peak NSM shear strength contribution $V_{f, k}^{\max }$ of: a) load step $\dot{\gamma}$; b) CDC inclination angle $\theta$; c) beam cross section depth $h_{w}$ and d) width $b_{w}$; e) strip cross section thickness $a_{f}$ and f) width $b_{f}$. 
Fig. 11. Influence on the peak NSM shear strength contribution $V_{f, k}^{\max }$ of: a) strips' spacing $s_{f}$; b) strips' inclination angle $\beta ;$ c) strip's Young's Modulus $E_{f} ; \mathrm{d}$ ) strip's strength $f_{f u} ;$ e) concrete compressive strength $f_{c m}$ and f) of the concrete fracture angle $\alpha$.

Fig. 12. Influence on the peak NSM shear strength contribution $V_{f, k}^{\max }$ of the local bond stress slip relationship parameters: a) $\tau_{0}$, b) $\tau_{1}$, c) $\tau_{2}$, d) $\delta_{1}$, e) $\delta_{2}$ and f) $\delta_{3}$. 


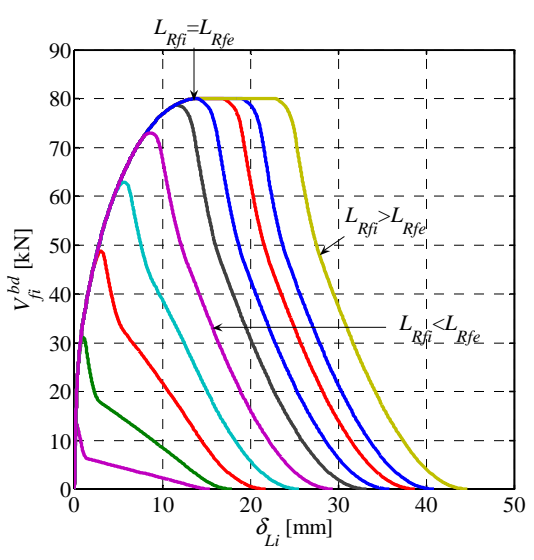

a)

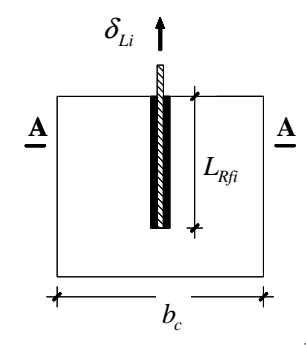

d)

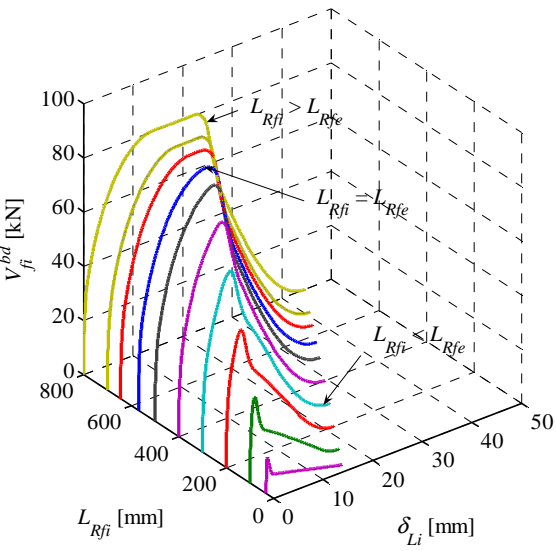

b)

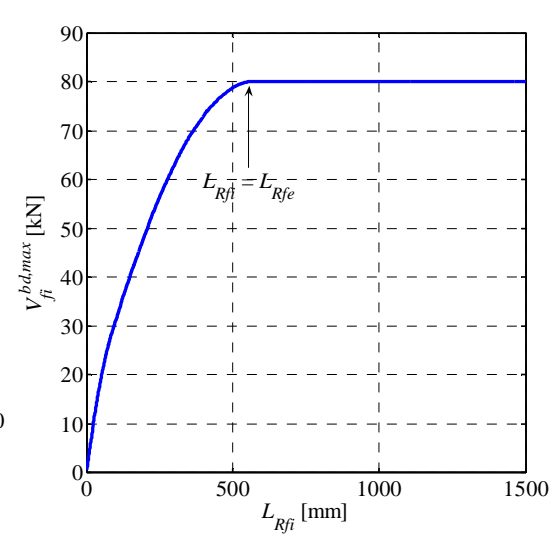

c)

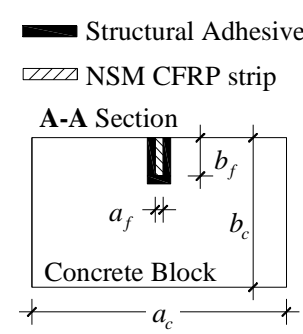

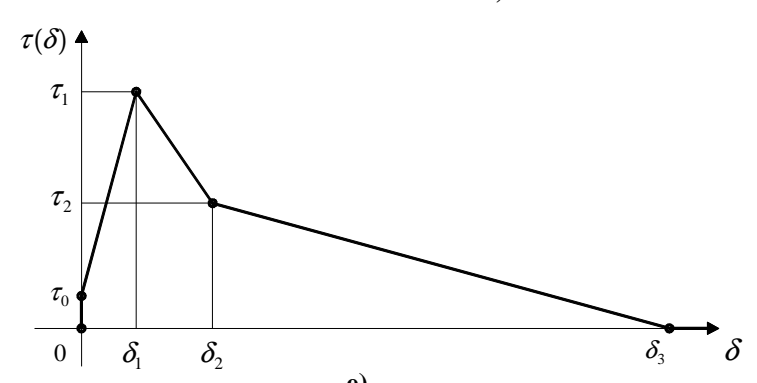

e)

Fig. 1. Bond-based behavior of a single Near Surface Mounted (NSM) FRP strip on a concrete prism: a-b) constitutive law $V_{f i}^{b d}\left(L_{R f i} ; \delta_{l i}\right)$ both in a bi-dimensional and in a three dimensional representation; c) dependence of the maximum bond-transferred force $V_{f i}^{b d, m a x}$ on the resisting bond length $L_{R f i}$; d) concrete prism with a single NSM FRP strip and e) adopted local bond stress-slip relationship. 

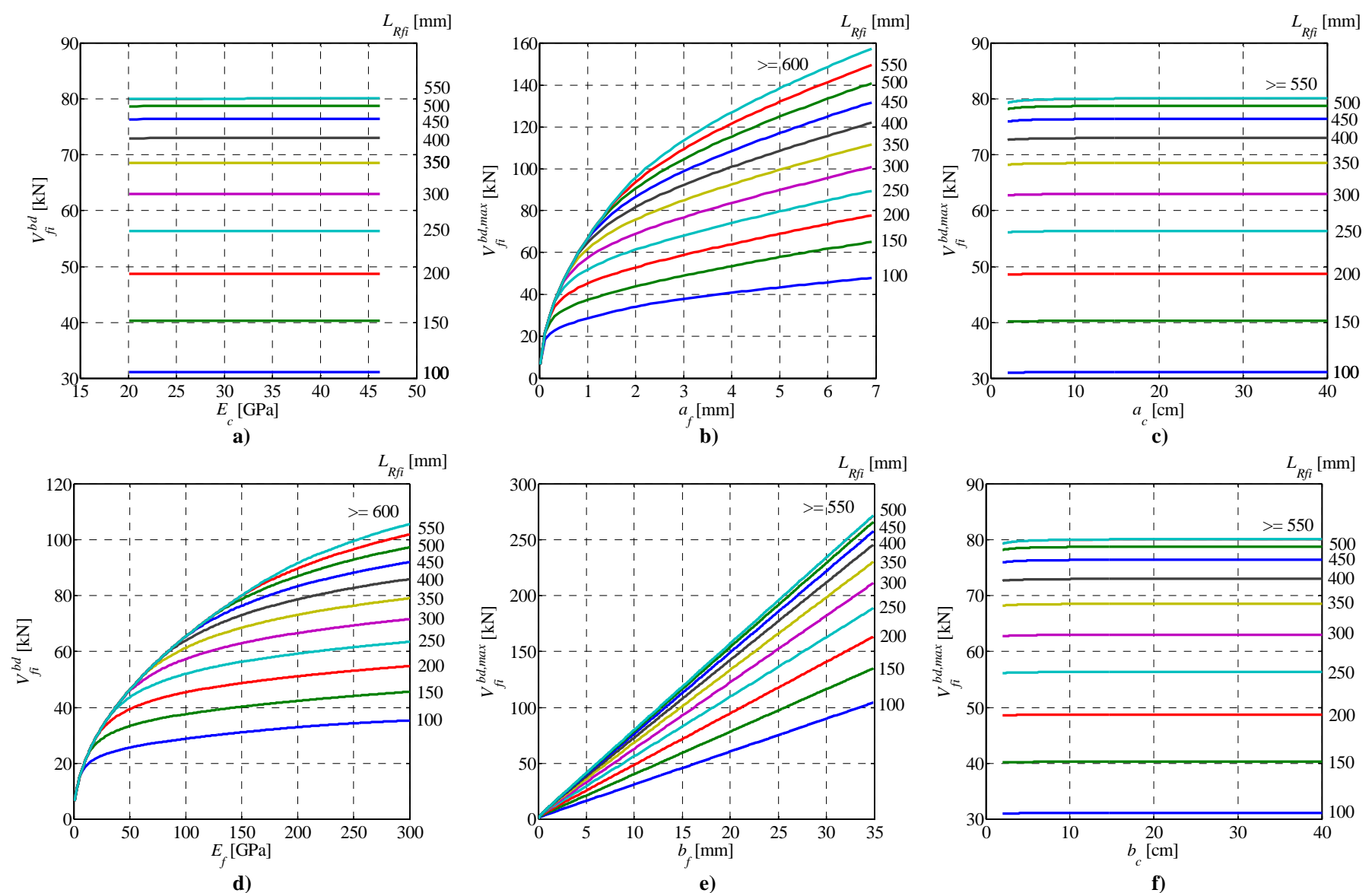

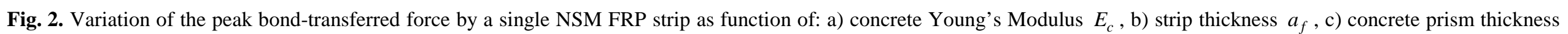
$a_{c}$, d) FRP Young's Modulus $E_{f}$, e) strip width and f) concrete prism width. 

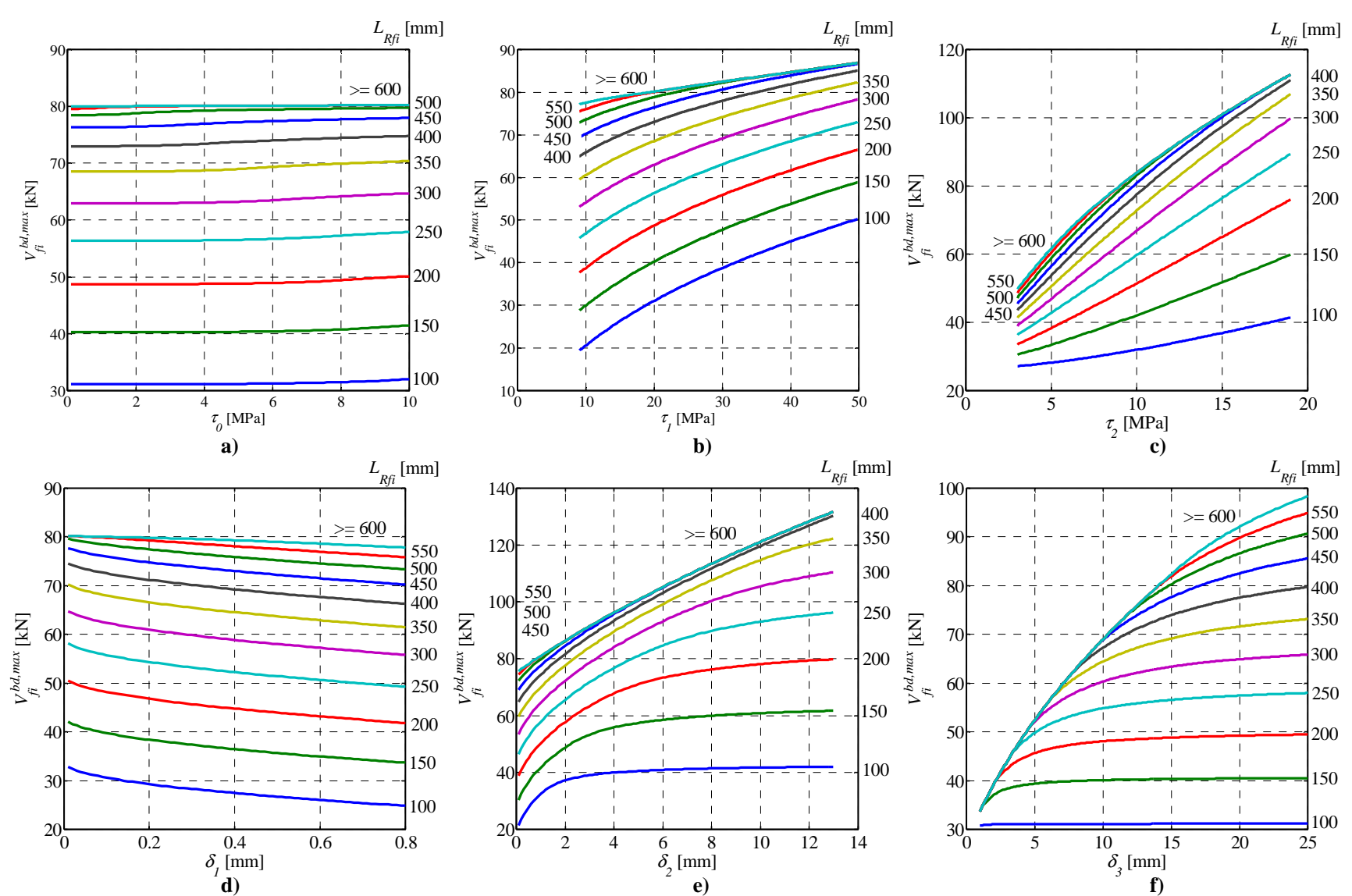

Fig. 3. Influence of the parameters defining the local bond stress-slip relationship on the peak force $V_{f i}^{b d, m a x}$ that a single NSM FRP strip can transfer, through bond stresses, to the surrounding concrete. 


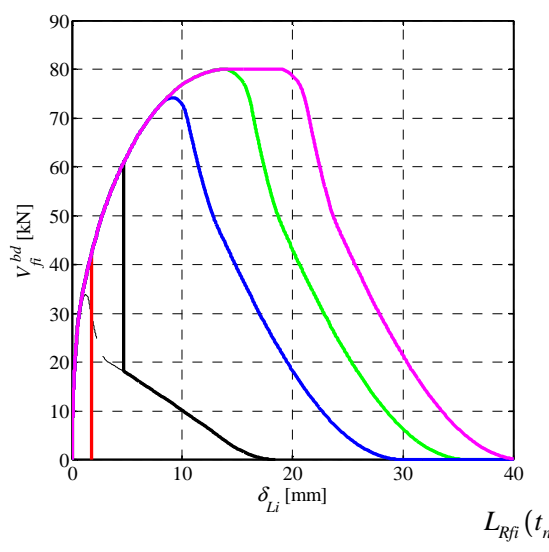

a)

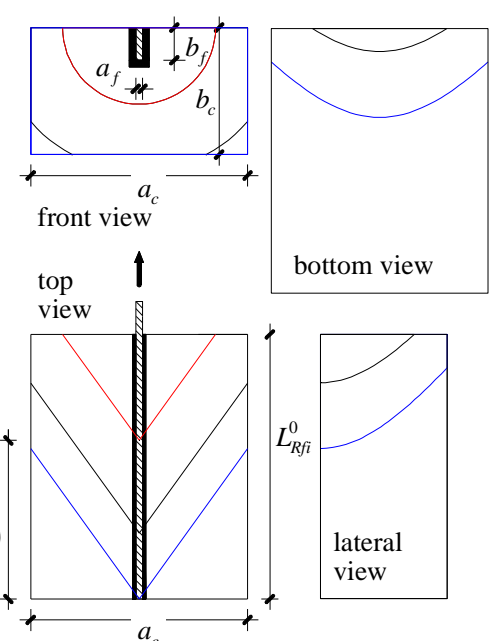

b)
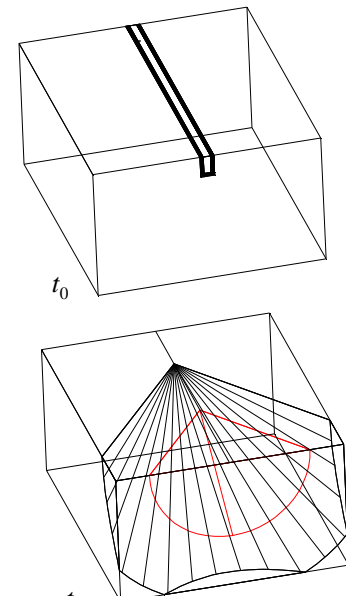
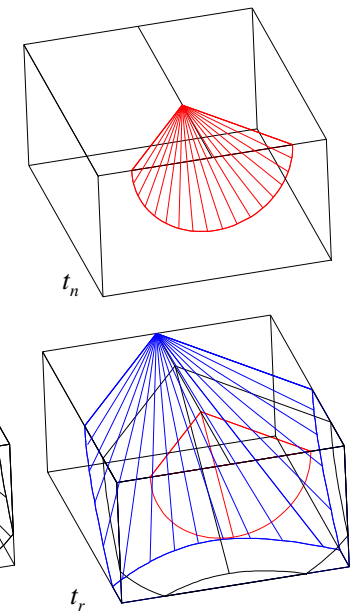

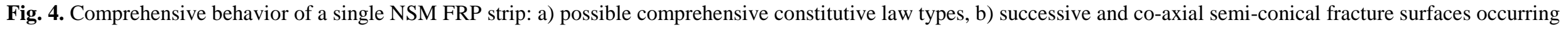
around the NSM strip and c) progressive reduction of the resisting bond length and penetration of the semi-conical fracture within the concrete prism. 

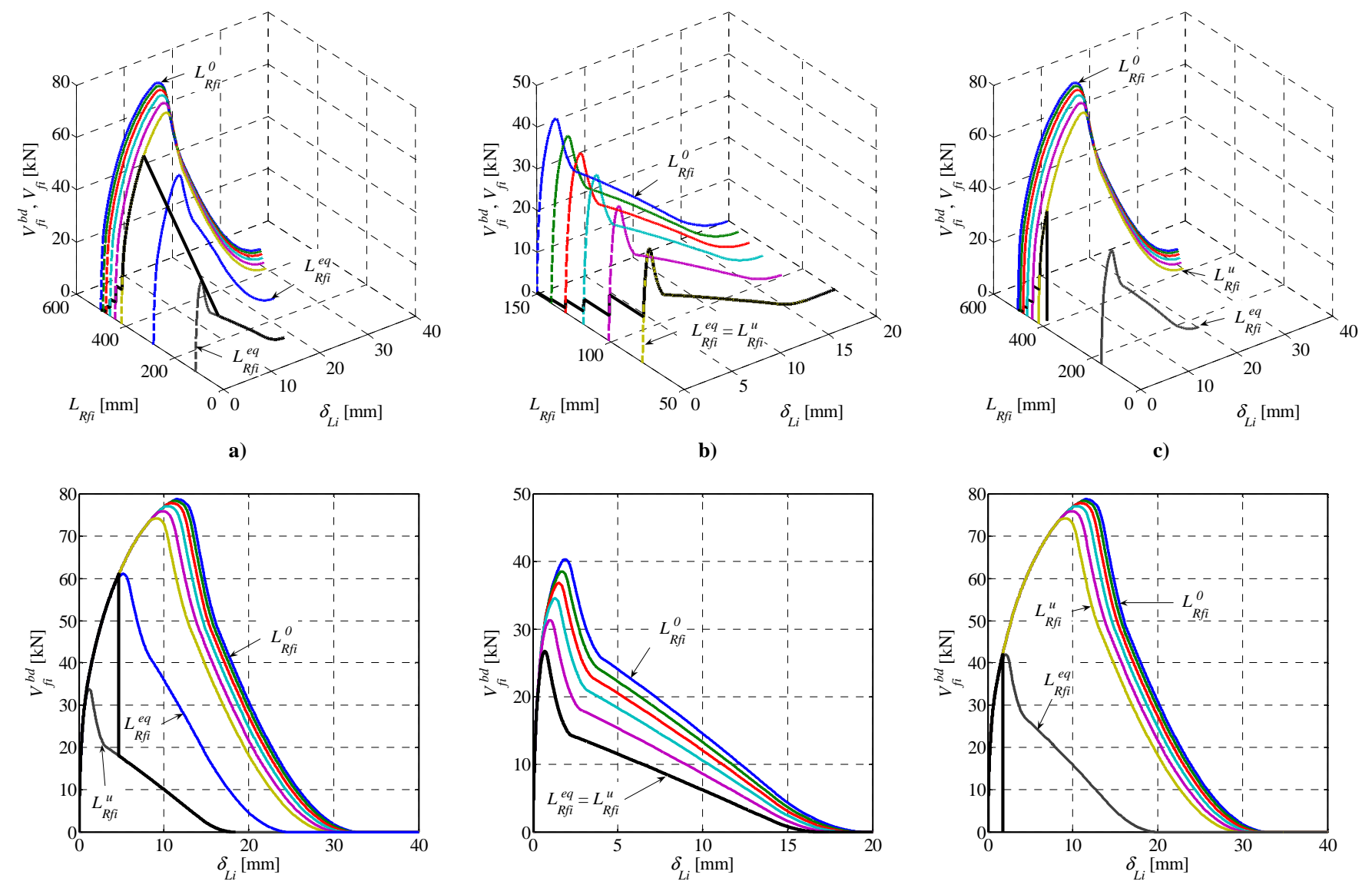

f)

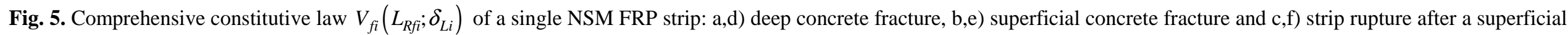
concrete fracture. 

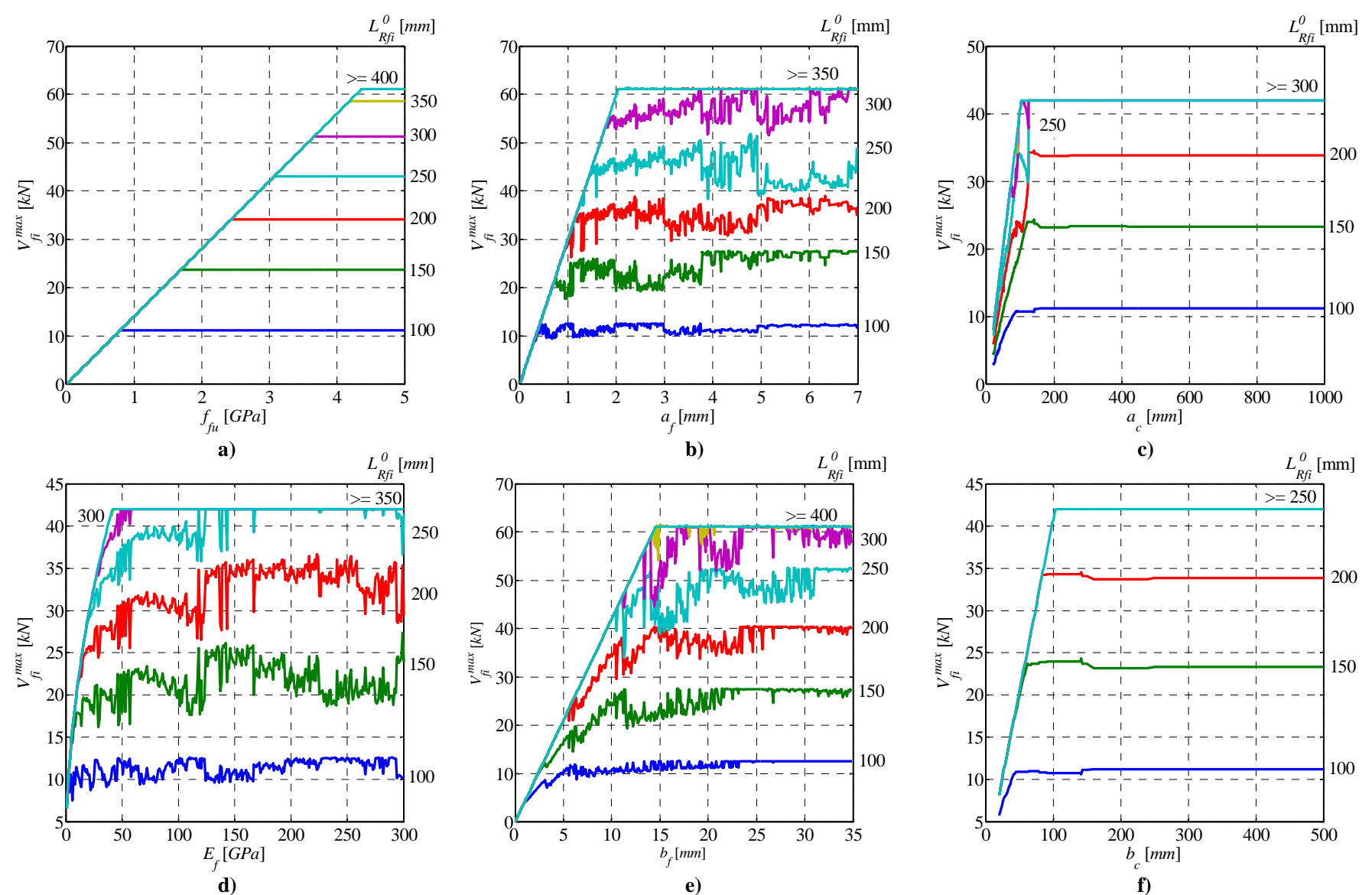

Fig. 6. Variation of the peak force that a single strip can comprehensively transfer to the surrounding concrete $V_{f i}^{\max }\left(L_{R f i}^{0}\right)$, as function of: FRP's a) tensile strength $f_{f u}$ and d) Young's Modulus $E_{f}$, strip cross section b) thickness $E_{f}$ and e) width $E_{f}$ and concrete prism cross section c) thickness $a_{c}$ and f) width $b_{c}$. 


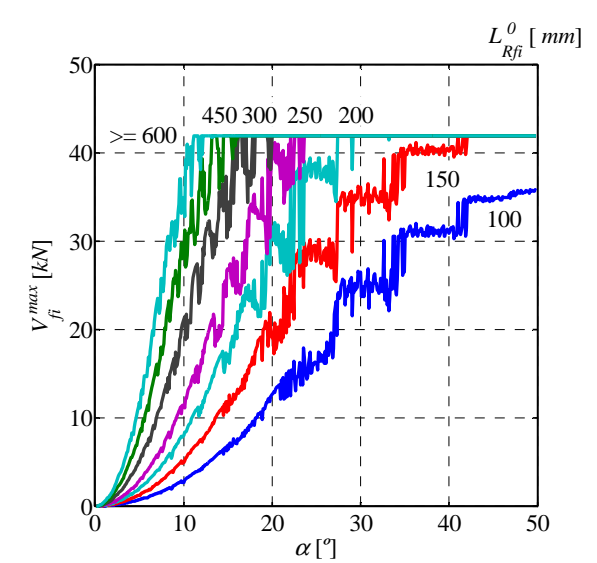

a)

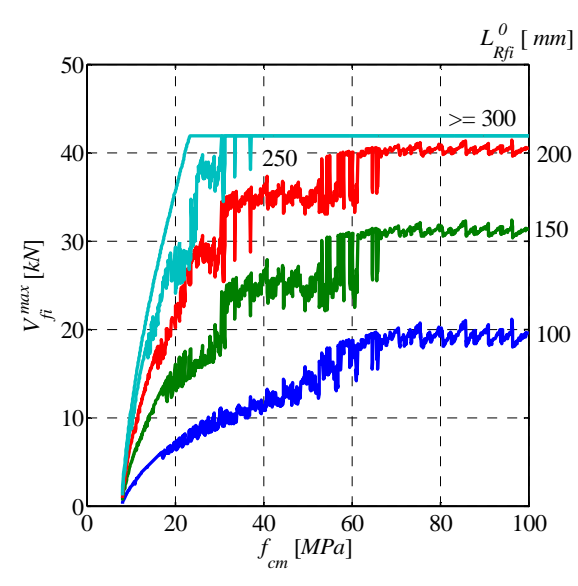

b)

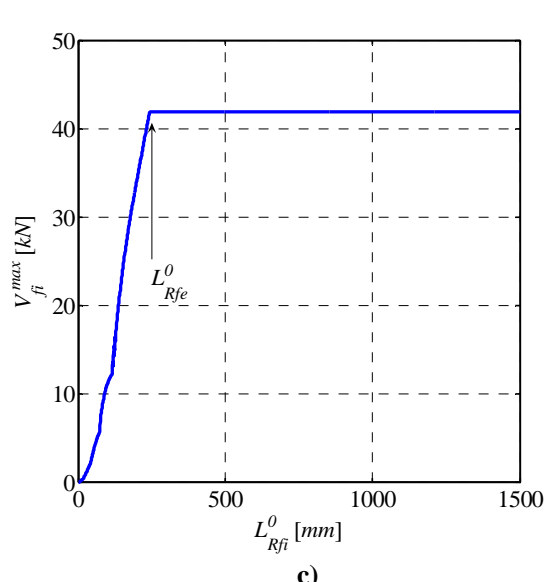

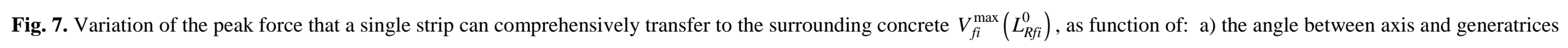

of the semi-conical concrete fracture surface $\alpha$, b) concrete average compressive strength $f_{c m}$ and c) initial resisting bond length $L_{R f i}^{0}$. 

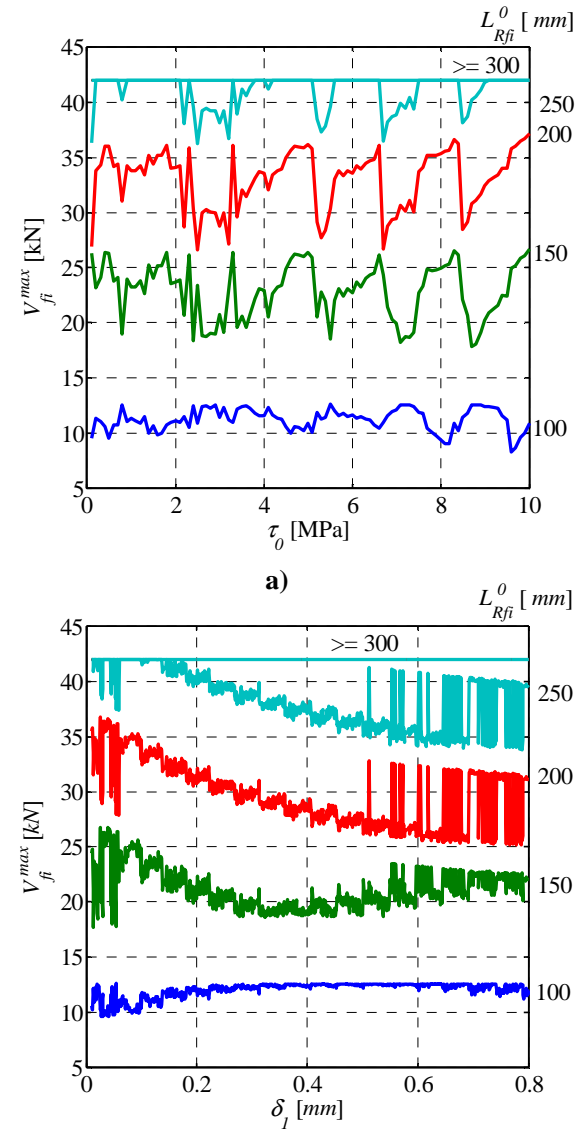

d)
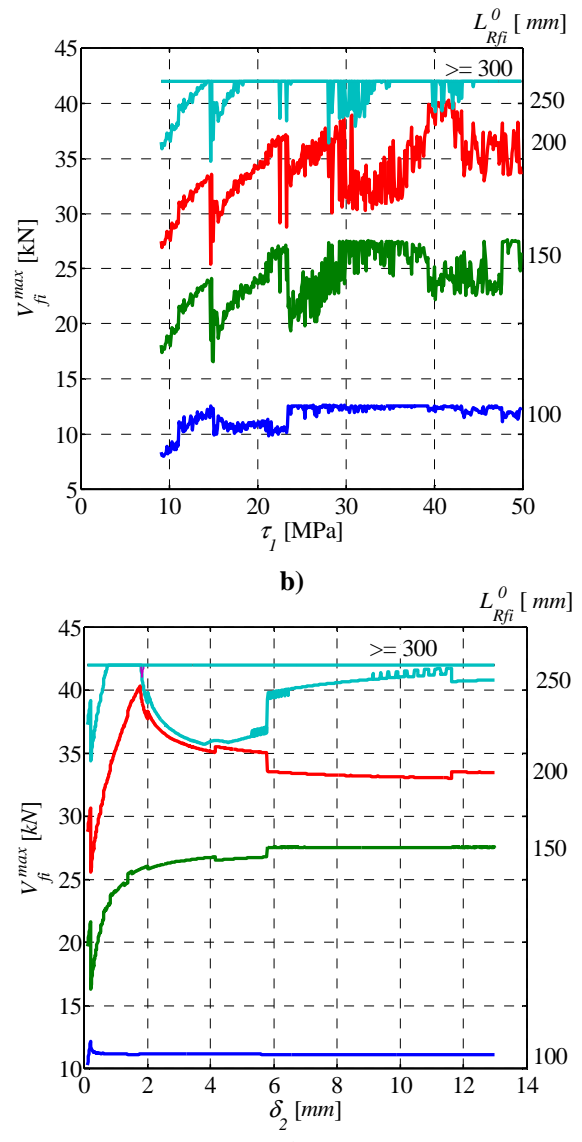
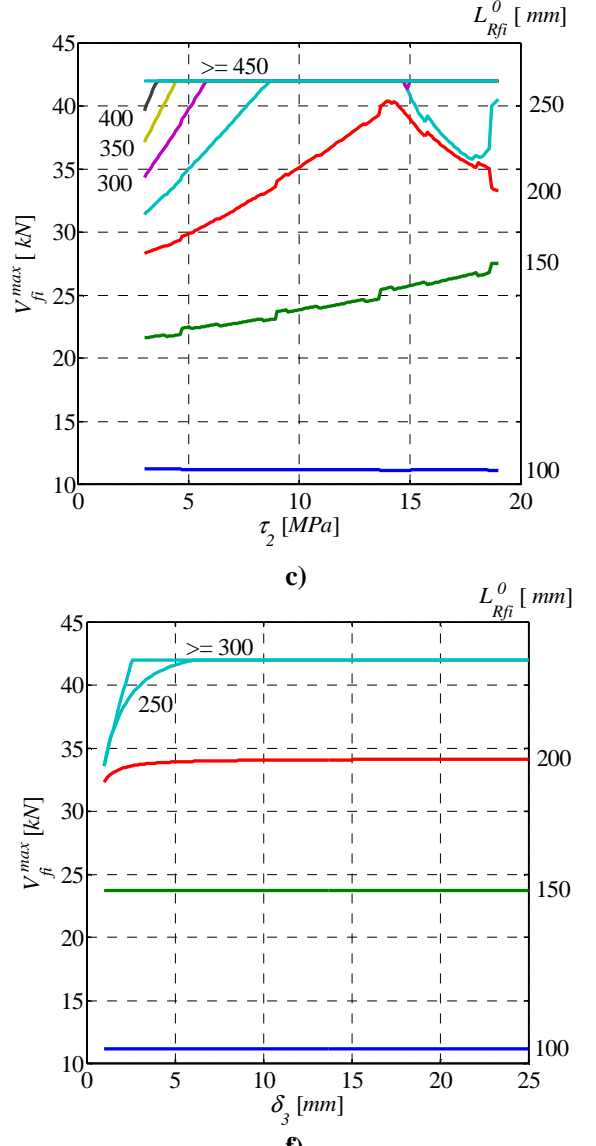

f)

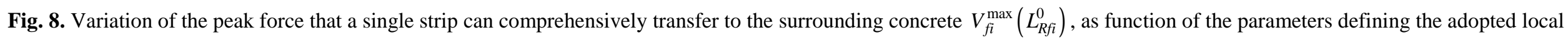
bond stress-slip relationship. 

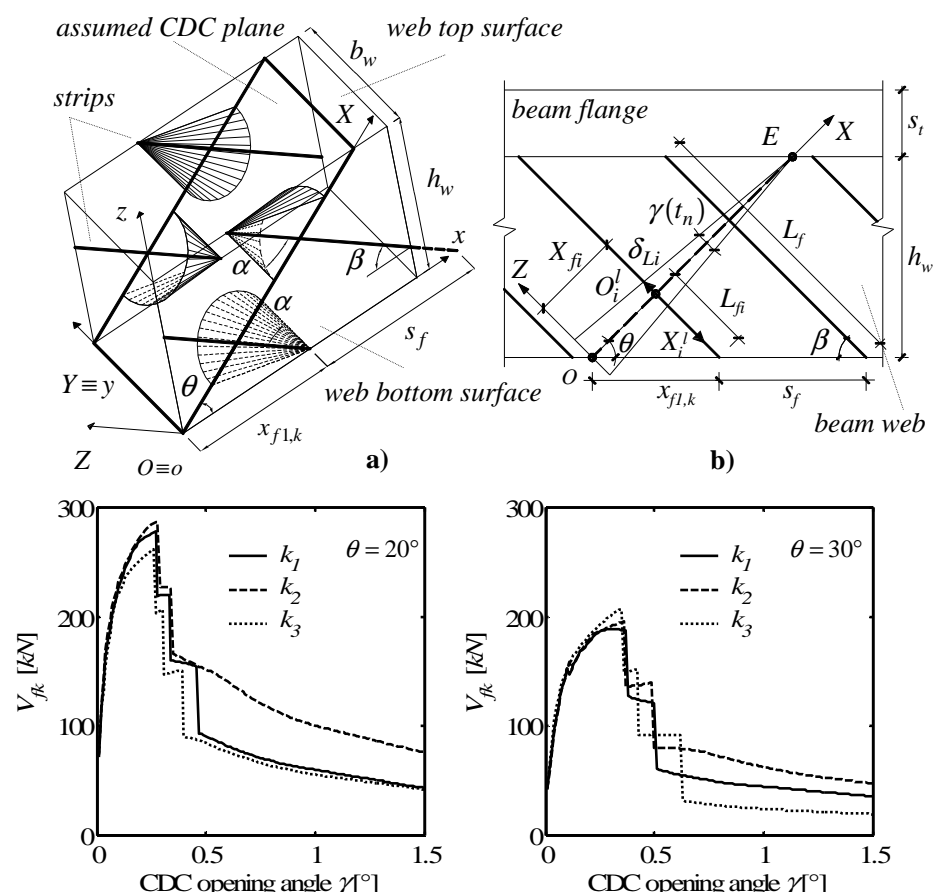

c)

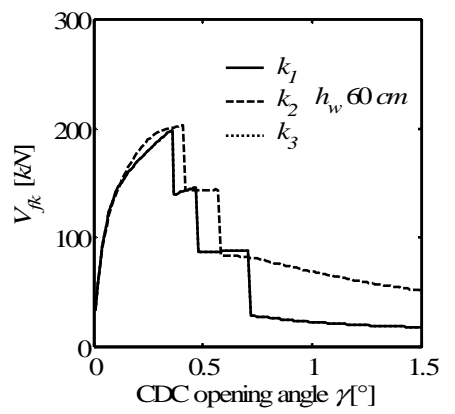

e)

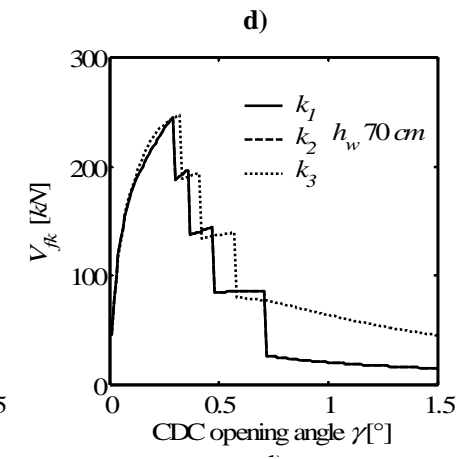

f)

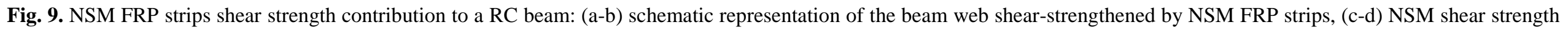
contribution to the adopted Reference Beam for two values of the CDC inclination angle and (e-f) for two values of the beam web depth. 

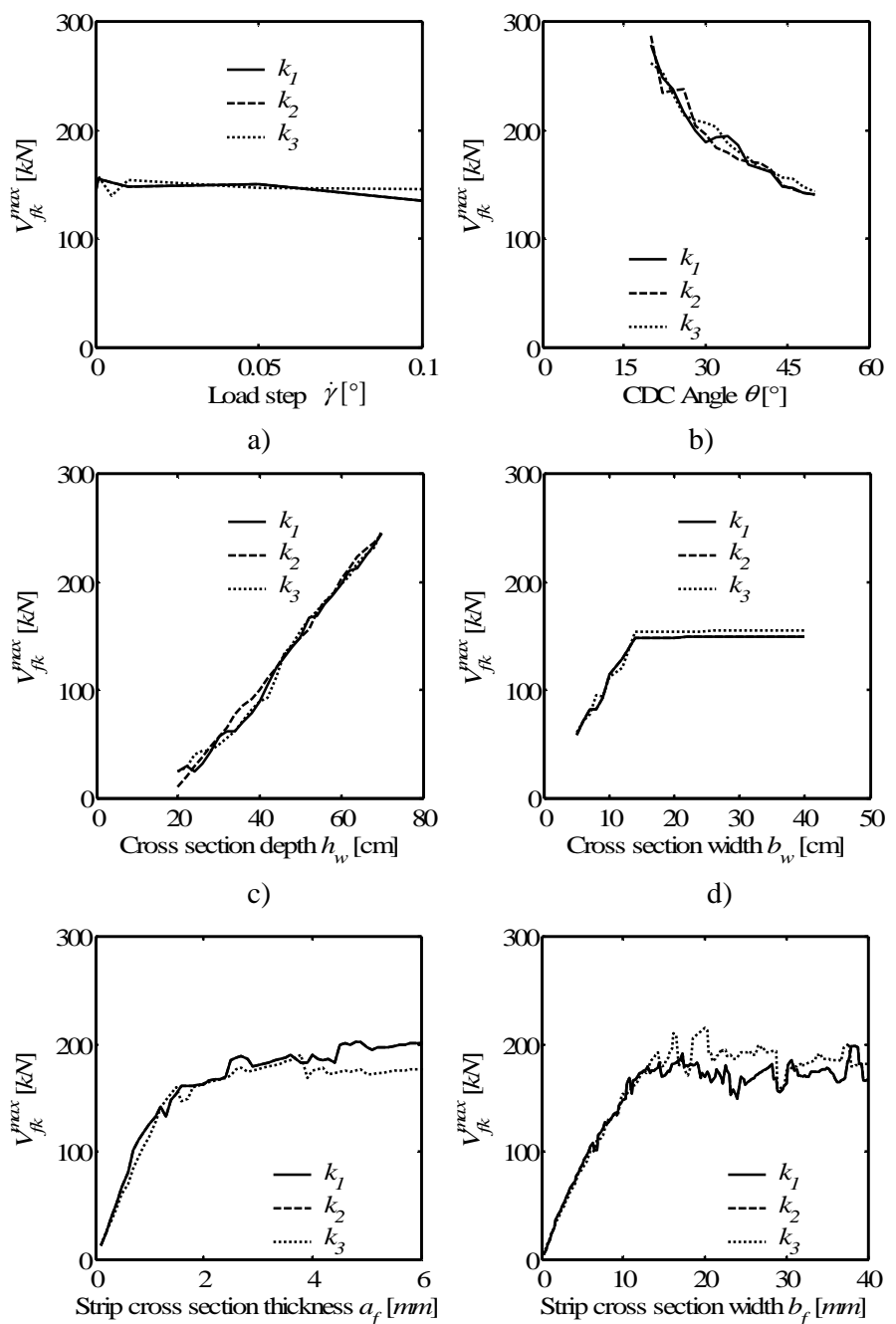

e)

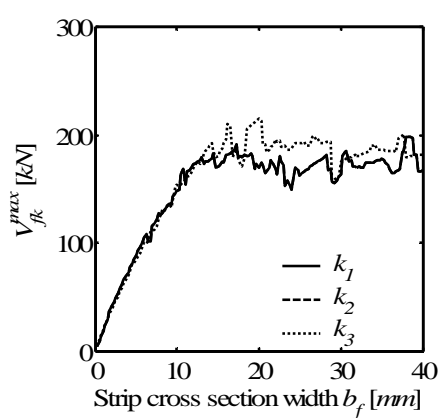

f)

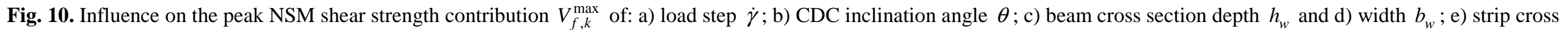
section thickness $a_{f}$ and f) width $b_{f}$. 

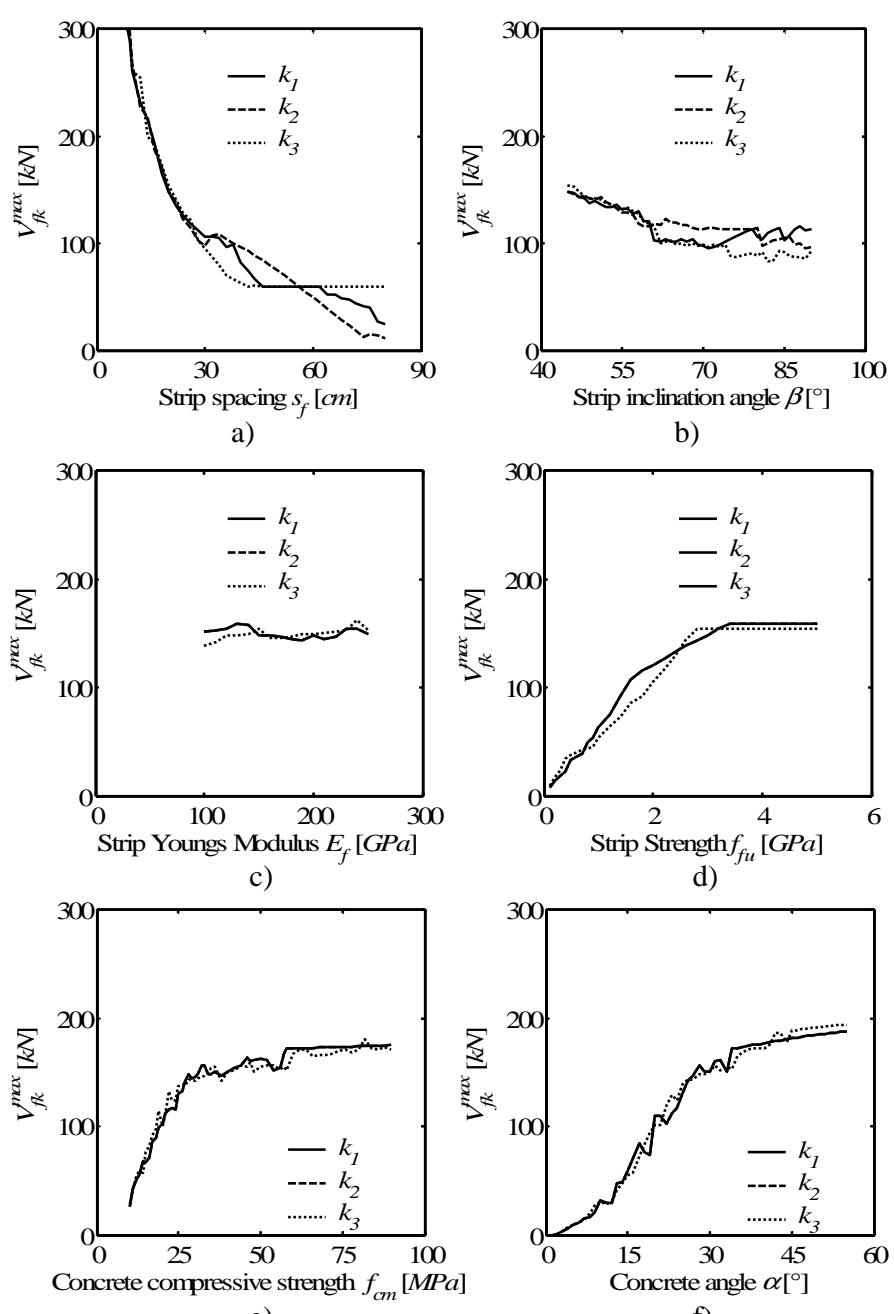

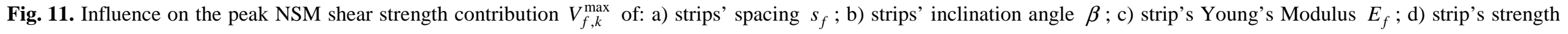
$f_{f u}$; e) concrete compressive strength $f_{c m}$ and f) of the concrete fracture angle $\alpha$. 

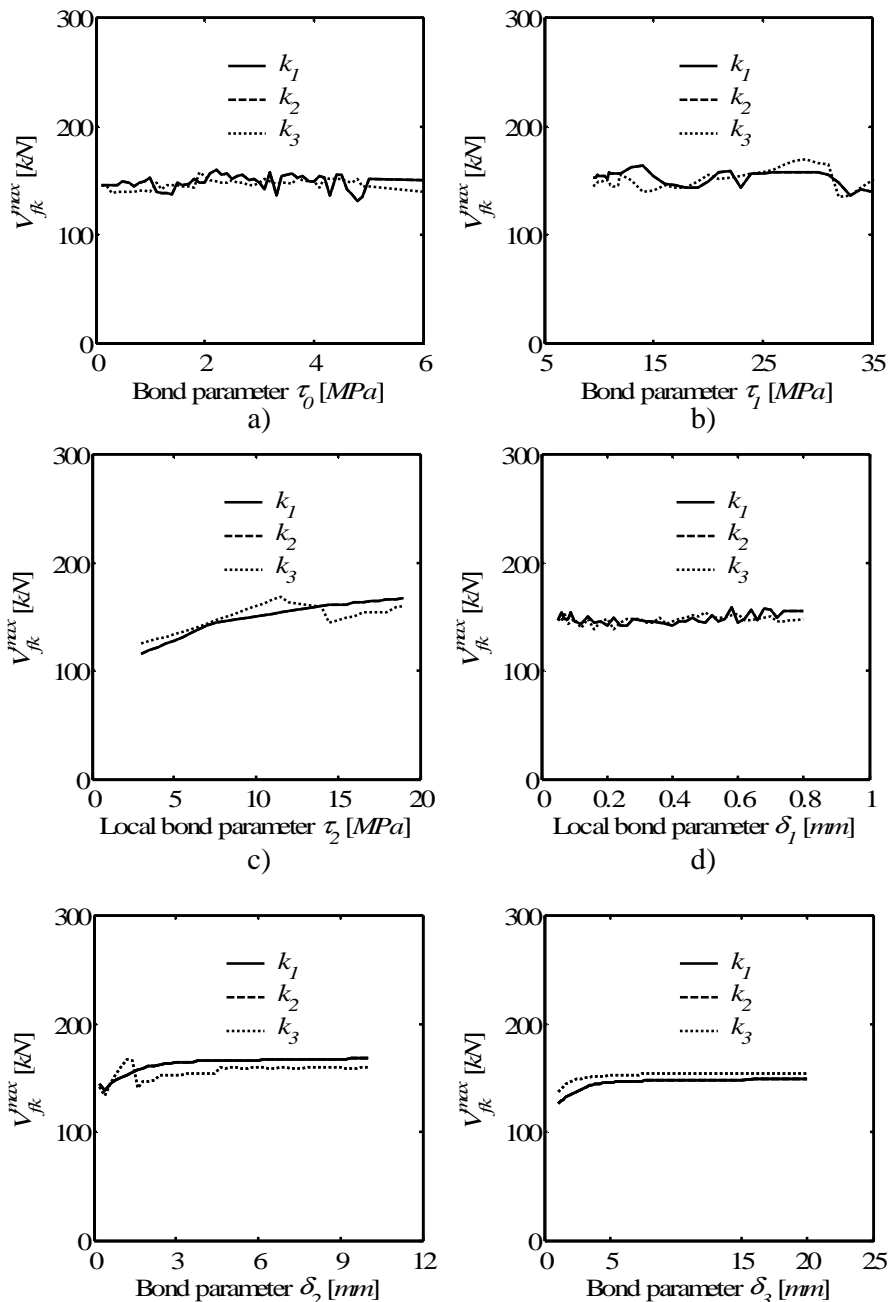

Fig. 12. Influence on the peak NSM shear strength contribution $V_{f, k}^{\max }$ of the local bond stress slip relationship parameters: a) $\tau_{0}$, b) $\tau_{1}$, c) $\tau_{2}$, d) $\delta_{1}$, e) $\delta_{2}$ and f) $\delta_{3}$. 